\title{
Geschichtsschreibung oder Rhetorik? Zum logos parakletikos bei Leon Diakonos
}

\author{
LARS HOFFMANN
}

Es ist beileibe kein Geheimnis, daß die byzantinische Historiographie zumindest partiell bestimmten rhetorischen Mustern und Gesetzen verhaftet ist und dabei antiken Vorbildern folgt. ${ }^{1}$ Die vorliegende Untersuchung will diesen Befund am Beispiel des logos parakletikos, ${ }^{2}$ der sogenannten Feldherrnrede, exemplifizieren, wie sie auch bei dem Historiker Leon Diakonos an einigen zentralen Punkten seines Geschichtswerks begegnet. ${ }^{3}$ Bereits Hans-Georg Beck und Herbert Hunger

1 Davon sprach schon H.-G. Beck, Zur byzantinischen „Mönchschronik“, in: C. Bauer/L. Boehm/ M. Müller (Hrsgg.), Speculum historiale. Geschichte im Spiegel von Geschichtsschreibung und Geschichtsdichtung, Freiburg i. Br./München 1965, 196-197 (= ders., Ideen und Realitäten in Byzanz. Gesammelte Aufsätze, London 1972, Nr. 16) in seiner Differenzierung der byzantinischen Chronistik; s. auch H. Hunger, The Classical Tradition in Byzantine Literature: the Importance of Rhetoric, in: M. Mullett/R. Scott (Hrsgg.), Byzantium and the Classical Tradition. Thirteenth Spring Symposium of Byzantine Studies, Birmingham 1981, 35-47, und ergänzend dazu auch R. Dostalova, Византийская историография (характер и формы), in: VV 43 (1982), bes. 22-26. Dem Thema „Geschichtsschreibung und Rhetorik“ ist auch auch der wichtige Band von U. Criscuolo/R. Maisano (Hrsgg.), Categorie linguistiche e concettuali della storiografia bizantina. Atti della quinta Giornata di studi bizantini, Napoli, 23-24 aprile 1998, Napoli 2000 , gewidmet, von dem weiter unten noch die Rede sein wird. Im übrigen bedanke ich mich für wertvolle Hinweise, Korrekturen und Ergänzungen bei meinen Kollegen Wolfram Brandes, Michael Featherstone, Michael Grünbart und John Haldon.

2 Zur Definition des $\lambda o ́ \gamma o \varsigma \pi \alpha \varrho \alpha \kappa \lambda \eta \tau \iota \kappa o ́ \varsigma$ sei vorab schon einmal verwiesen auf J. Albertus, Die $\Pi \alpha \varrho \alpha \kappa \lambda \eta \tau \iota \kappa o$ in der griechischen und römischen Literatur. Straßburg 1908, auf dessen Begrifflichkeit im folgenden noch näher einzugehen sein wird. Hilfreich für die Auseinandersetzung mit dem Thema ist auch R. Leimbach, Militärische Musterrhetorik. Eine Untersuchung zu den Feldherrnreden des Thukydides, Stuttgart 1985, obwohl diese Abhandlung in klassischphilologischer Manier eher die überlieferten Reden kommentiert als sie unter formalrhetorischen Gesichtspunkten näher einzuordnen, was Albertus intendiert hatte. Dessen Leistung erkannte O. Luschnat, Die Feldherrnreden im Geschichtswerk des Thukydides (Philologus, Supplementband 34/2), Leipzig 1942, 1, durchaus an, obwohl er seinerseits bei Albertus das Fehlen eines detaillierten Kommentars bemängelte, ohne mit diesem Tadel jedoch dem Leitinteresse seines Vorgängers gerecht zu werden.

3 A. Markopoulos, Byzantine History Writing at the End of the First Millennium, in: P. Magdalino (Hrsg.), Byzantium in the Year 1000 (The Medieval Mediterranean 45), Leiden/Boston 2003, 190, bescheinigt Leon Diakonos teilweise wohl zu Recht (obwohl dessen Stil und Sprache in der Tat recht weit von Chronisten wie Theophanes entfernt sind!), daß sein Text im Gegensatz zur offiziellen Geschichtsschreibung nicht mit höfischer Rhetorik überfrachtet sei, und nennt da- 
hatten auf diesen Sachverhalt hingewiesen. Während sich jedoch Beck unter der Maßgabe seines eigenen Themas auf einige inhaltliche Gesichtspunkte der Reden beschränkte, ${ }^{4}$ gewinnt man bei Hunger den Eindruck, Leon Diakonos habe diese kurzen Passagen gleichsam mit dem rhetorischen Pfefferstreuer über sein Werk verteilt, ${ }^{5}$ um auf diese Weise einem Text mehr Würze zu geben, dem bereits Karl Krumbacher - ob nun zu Recht oder zu Unrecht, bleibt an dieser Stelle ohne Belang - sprachliche Mängel in jeder Hinsicht bescheinigt hatte. ${ }^{6}$ Sicherlich gehört das Werk nicht zu den bedeutendsten Erzeugnissen der byzantinischen Historiographie, ${ }^{7}$ und Hunger bemerkte durchaus zutreffend, die Chronistik der Byzanti-

für biographische Gründe. Aber das bedeutet ja nicht, daß die traditionellen rhetorischen Elemente im Sinne von Hermogenes oder Lukianos (worauf weiter unten noch einzugehen sein wird) damit gleichfalls ausfallen würden! Allerdings erlaubt der räumliche Abstand vom kaiserlichen Umfeld, einen Text nach eigenem, respektive erlerntem Geschmack zu verfassen, wenngleich auch in diesem Fall der öffentliche Vortrag intendiert gewesen sein muß, was zu große Freiheiten in der sprachlichen Gestaltung verhinderte.

4 H.-G. Beck, Res Publica Romana. Vom Staatsdenken der Byzantiner, München 1970, 22-23. Allerdings läßt die knappe, treffsichere Charakterisierung der hier zu behandelnden Textstücke durch Beck bereits erkennen, daß ihm die formale und inhaltliche Zweckhaftigkeit der Redeeinschübe durchaus bewußt war.

5 H. Hunger, Die hochsprachliche profane Literatur der Byzantiner (HdAW XII, Byzantinisches Handbuch 5, 1-2), München 1978, II 369-370 (mit Anm. 189, doch wird man bei Leon die von Hunger angegebenen Bücher XIII und XLII vergeblich suchen: Es handelt sich dabei um Seitenzahlen, so daß es zu einer Doppelzitierung kam).

6 K. Krumbacher, Geschichte der byzantinischen Litteratur von Justinian bis zum Ende des oströmischen Reiches (527-1453), München ${ }^{2} 1897$, bes. 267-268. Leon ist für ihn ein Musterbeispiel des „halbgebildeten Byzantiners“, der sich in treuer Agathias-Mimesis u. a. auf unerträglich langweilige Satzkonstruktionen beschränkt. Immerhin teilte Hunger, Hochsprachliche profane Literatur (s. Anm. 5) diese Sicht der Dinge nicht, wobei er Sprache und Stil unseres Historikers weder besonders hervorhebt, noch kritisiert oder abqualifiziert. Funktion und Zweck der von Hunger im Anschluß an Beck, Mönchschronik (s. Anm. 1), 197 aufgeführten, eher chronistischen Elemente wären aber im Einzelfall zu überprüfen. Krumbachers These von der Nachahmung des Agathias, die Hunger, Hochsprachliche profane Literatur (s. Anm. 5), I, 370, bereits relativierte und die auf die Kommentierung der Edition durch C. B. Hase zurückgeht (s. dort etwa 397: „Leo Agathiam, ut toto opere, item hic imitatur“; 417: „По $\lambda \varepsilon \mu$ í $\alpha$ Agathias,

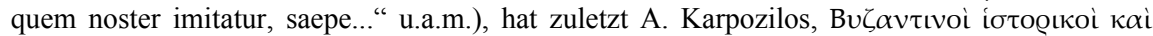

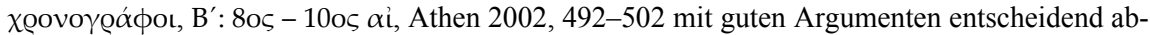
schwächen können. Eine gründliche Neubewertung des gesamten Textes ergäbe sich aber anhand der Ausführungen von P. Magdalino, A History of Byzantine Literature for Historians, in: P. Odorico/P. A. Agapitos (éd.), Pour une «Nouvelle» histoire de la Littérature byzantine. Actes du Colloque international philologique, Nicosie 25-28 mai 2000 (Dossiers byzantins 1), Paris 2001, bes. 168-170, denn Leon Diakonos ist ein gutes Beispiel dafür, zu welchen Mißverständnissen eine unbedarfte Lektüre des Textes führen kann, die rhetorische und sprachlichkompositorische Elemente ignoriert (s. dazu u. 117).

7 Literatur wird nicht dadurch wertvoll, daß sich der moderne Byzantinist damit beschäftigt und demgemäß auf Hör- und Lesegewohnheiten der Byzantiner anspielt, wie dies etwa J. Ljubarskij, How Should a Byzantine Text Be Read?, in: E. Jeffreys (Hrsg.), Rhetoric in Byzantium. Papers from the Thirty-fifth Spring Symposium, Exeter-College, University of Oxford, March 2001, Alders-hot 2003, 118-120, formulierte. 
ner habe insofern Einfluß auf den Text genommen, daß man bei Leon auf einzelne, der antiken Geschichtsschreibung gattungsfremde Elemente stieße. ${ }^{8}$

Dies führt zu der Frage, ob man überhaupt zwischen einer byzantinischen Weltchronistik und einer byzantinischen Historiographie differenzieren soll. Beck hatte dies bereits bestritten, ${ }^{9}$ und für die erstgenannte sollte man mit Elizabeth Jeffreys, die zu Recht auf die religiöse Zuspitzung und strenge Verchristlichung des von Sextus Iulius Africanus entwickelten chronographischen Konzeptes durch Eusebios von Kaisareia hinwies, ${ }^{10}$ das Epitheton „byzantinisch“ aus sachlichen Gründen besser durch „,christlich“ ersetzen, war es doch die neue Religion mit ihrem universalistischen Anspruch, die eine solche Literaturgattung überhaupt erst hervorbringen konnte, ${ }^{11}$ da sie ihrerseits einen fixen, gottgewirkten Anfangs-

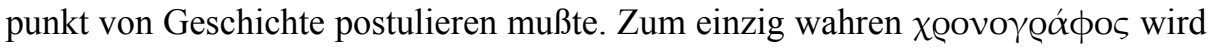
vor dem Hintergrund einer solchen Konzeption Gott selbst: Er schreibt Tag um Tag die Geschichte, die von Seiten seiner Schöpfung nur noch rein passiv aufgezeichnet werden kann. ${ }^{12}$ Streng genommen ist ein Verfasser solcher Texte nichts

8 Hunger, Hochsprachliche profane Literatur (s. Anm. 5), I 370. Allerdings sollte man zu den dort aufgezählten Merkmalen auch den sehr unvermittelten Einstieg des Textes hinzunehmen, der nach einem knappen, mit apokalyptischen Anspielungen versehenen Prooimium mit einer exakten Datierung des Todestags von Kaiser Konstantin VII. im Stile der Chronistik einsetzt. In der Beurteilung ähnlich J. Ljubarskij, How Should a Byzantine Text Be Read? (s. Anm. 7), 119 und 120.

9 Beck, Mönchschronik (s. Anm. 1), 196-197; s. aber auch Hunger, Hochsprachliche profane Literatur (s. Anm. 5), I bes. 254 und 372, und A. Kazhdan, Der Mensch in der byzantinischen Literaturgeschichte, in: JÖB 28 (1979) 3-5.

10 E. Jeffreys, The Attitudes of Byzantine Chroniclers Towards Ancient History, in: Byz 49 (1979) 215-216. Dies erscheint um so berechtigter, da dieses Konzept durch die Vermittlung des Hieronymus ja auch im lateinischen Westen zu einer großen weltchronistischen Literatur führte.

11 An dieser Stelle kann der eher zwanglose Umgang nicht behandelt werden, mit dem die byzan-

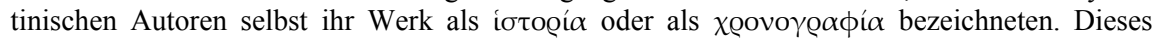
Thema wurde überdies schon häufig genug behandelt. Dennoch gilt es hier den eindeutigen Hinweis bei Joannes Skylitzes, Historia, 3, 26-4, 35 (Thurn) zu beachten, der Leon zu denjeni-

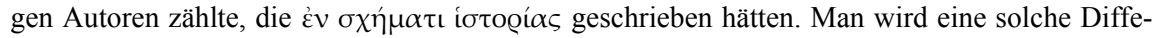
renzierung allenfalls auf eine vergleichende Analyse einzelner Texte beschränken können, wie dies etwa D. E. Afinogenov, Some Observations on Genres of Byzantine History, in: Byz 62 (1992) 13-33 für Georgios Monachos und Joannes Zonaras getan hat.

12 Hungers Charakterisierung der Chronistik als „Trivialliteratur“ (Hochsprachliche profane Literatur [s. Anm. 5], I, hier bes. 257-258), die bei ihm terminologisch auf das entsprechende Lemma im „Großen Brockhaus“ des Jahres 1974 zurückgeht und seither öfter aufgegriffen wurde, ist gerade vor einem solchen Hintergrund unangemessen und abzulehnen - was bereits Kazhdan, Der Mensch (s. Anm. 9), 2 erkennen ließ (ergänzend auch J. N. Ljubarskij, New Trends in the Study of Byzantine History, in: DOP 47 (1993) 135 = ders., Византийские историки и писатели, Sankt Petersburg 1999, 312). Könnte man denn nicht auch einige der scheinbar rein persönlichen Beweggründe, die Michael Psellos zu seiner Chronographia führten (entsprechende Textbeispiele bei R. Macrides, The Historian in the History, in: C. N. Constantinides [et al.] [Hrsgg.], ФI $\Lambda \mathrm{E} \Lambda \Lambda \mathrm{HN}$. Studies in Honour of Robert Browning, Venedig 1996, 212-215; zur Bewertung des Psellos s. aber auch J. N. Ljubarskij, Михаил Пселлю Личностъ и творчество. 
weiter als ein ausführendes Organ, da er einzig die verschiedenen Manifestationen des von ihm beobachteten, unabänderlichen göttlichen Willens niederschreibt. Die Weltchronistik verbietet es demnach, etwa einen bestimmten Kaiser in den Mittelpunkt der schriftstellerischen Bemühungen zu stellen, so daß sich der für alle Menschen gleiche Jahreszyklus als wertfreies Gliederungsschema gleichsam aufdrängt. ${ }^{13}$

Die Geschichtsschreibung bietet in dieser Hinsicht wesentlich mehr Spielraum, auch wenn sie ihrerseits die Erwartungen eines Publikums zu befriedigen hat, das mehr oder weniger rhetorisch und literarisch gebildet ist. Der Autor und seine Leser- bzw. Hörerschaft stehen demnach in einem spielerischen $\alpha$ z $\gamma \omega v$, den ersterer durch die Wahl eines geeigneten Themas sowie durch sprachliche Kunstfertigkeit für sich entscheiden konnte. ${ }^{14}$ Dafür war es wiederum notwendig, daß sich ein solcher Autor auch tatsächlich zu erkennen gab. Mit Leon Diakonos trat im ausgehenden 10. Jahrhundert ein solcher Mann klar hervor, worauf bereits Jakuv N. Ljubarskij hinwies: Im Gegensatz zu seinen Vorgängern aus den sogenannten „dunklen Jahrhunderten“ gibt Leon die Anonymität auf, wie dies im

К истории византийского предгуманизма, Moskau 1978, 185-202), als ebenso trivial bezeichnen - oder gar die Motivation einer sich um die Herrschaft betrogen fühlenden Anna Komnene, endlich einmal die Dinge so aufzuzeigen, wie sie „,wirklich“ waren (s. etwa Alexias I, 1, 2; I 2, 2; I 2, 3, 45-46 u.a.m [Reinsch])? Ergänzend zu letzterer auch Dostalova, Византийская историография (s. Anm. 1), 27. Allerdings wäre eine solche Charakterisierung in beiden Fällen unangemessen, da sie die Intention der Werke verfehlt.

13 Die unersprießliche und schon häufig geführte Diskussion „Byzantinische Geschichtsschreibung vs. Chronistik“, die bereits J. Karayannopulos/G. Weiss, Quellenkunde zur Geschichte von Byzanz (324-1453) (Schriften zur Geistesgeschichte des östlichen Europa 14), Wiesbaden 1982, I 65-66, mit guten Gründen aufgegeben haben, kann und soll hier natürlich nicht wiedergegeben werden. Die dort gegebene Definition der Chroniken als eine „Weltgeschichte mit apokalyptischem Hintergrund" trifft den Sachverhalt aber sehr genau - was nicht verwundert, denn als Schüler des Erlanger Theologen E. Stauffer besaß G. Weiß auch ein sicheres Gespür für theologisch-apokalyptische Elemente in einem Text; vgl. auch P. Magdalino, The History of the Future and Its Uses: Prophecy, Policy and Propaganda, in: R. Beaton/Ch. Roueché (Hrsgg.), The Making of Byzantine History. Studies dedicated to Donald M. Nicol, Cambridge 1993, 25-26, sowie W. Brandes, Liudprand von Cremona (Legatio Cap. 39-41) und eine bisher unbeachtete Korrespondenz über die Bedeutung des Jahres 1000 a.D., in: BZ 93 (2000) 454-456 und bes. 463.

14 Dazu P. Magdalino, The Empire of Manuel I Komnenos, 1143-1180, Cambridge 1993, 339-

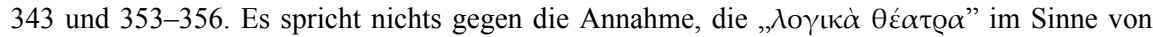
M. Mullett, Aristocracy and Patronage in the Literary Circles of Comnenian Constantinople, in: M. Angold (Hrsg.), The Byzantine Aristocracy IX-XIII Centuries (BAR, International Series, 221), Oxford 1984, hier bes. 174-180, auch schon für die Zeit des Leon Diakonos anzusetzen, wenn auch die entsprechenden literarischen Zeugnisse erst in die Komnenenzeit gehören. Dazu vgl. auch St. Efthymiades, L'enseignement secondaire à Constantinople pendant les $\mathrm{XI}^{\mathrm{e}}$ et XII ${ }^{\mathrm{e}}$ siècles: modèle éducatif pour la Terre d'Otrante au XIII ${ }^{\mathrm{e}}$ siècle, in: Nea Rhome 2 (2005) 261, sowie G. Cavallo, A Bisanzio. Pratiche intelletuali e modelli del passato, in: J.-M. Sansterre, L'autorité du passé dans les sociétés médiévales (Collection de l'École Française de Rome 333 $=$ Institute Historique Belge de Rome 52), Brüssel/Rom 2004, 336-337. 
Gefolge auch viele andere Geschichtsschreiber tun sollten. ${ }^{15} \mathrm{Da}$ es ihm dabei nicht etwa um Selbstdarstellung geht, versteht sich von selbst, und wird auch aus dem Werk klar, dem Leon durch seine wenigstens partiell angedeutete persönliche Teilnahme an den von ihm berichteten Vorgängen eine höhere Glaubwürdigkeit verleihen möchte. ${ }^{16}$ Und rhetorische Stilmittel, die den Protagonisten eines beschriebenen Vorgangs als aktiven Handlungsträger erkennen lassen, verstärken einerseits eben diese Glaubwürdigkeit, um andererseits auch die Person des Autors als Berichterstatter wieder in den Hintergrund zu rücken. ${ }^{17}$

Die direkte Rede im formalen Gewand des logos parakletikos ist nun eine dieser Möglichkeiten. Formal gehört dieser logos als beratende Rede zum genos demegorikon, das nach Anaximenes, ${ }^{18}$ Aristoteles ${ }^{19}$ und Hermogenes einem Katalog von bis zu sechs Kriterien genügen müsse: Der vermittelte Inhalt müsse ge-

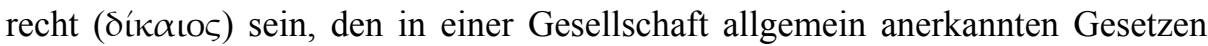
und Regularien entsprechen (vó $\mu \mu о \varsigma$ ), dasjenige befördern, was einer Gesellschaft oder Gruppe nützt ( $\sigma u ́ \mu \phi \varepsilon @ o v)$, weiterhin auch am Guten ausgerichtet sein

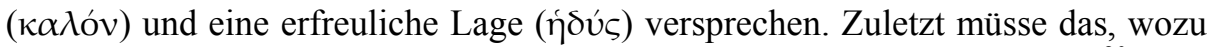
geraten oder wovon auch abgeraten wird, leicht umsetzbar (@ó́dı

15 J. N. Ljubarskij, "Writers Intrusion" in Early Byzantine Literature, in: XVIII" Congrès International des études byzantines. Rapports pléniers, Moskau 1991, 441 (zu Leon) sowie 442-444 (zu Michael Attaleiates und Michael Psellos) [der Artikel wurde unverändert nachgedruckt in: ders., Византийские историки и писатели, Sankt Petersburg 1999, 338-354]; ergänzend natürlich R. Scott, The Classical Tradition in Byzantine Historiography, in: M. Mullett/R. Scott (Hrsgg.), Byzantium and the Classical Tradition, Birmingham 1981, 61-74 sowie R. Macrides, The Historian in the History (s. Anm. 12), bes. 206-210 und 223-224. Leon gehört dabei aber zu den Autoren, die - worauf Macrides abschließend hinweist - mit ihrer persönlichen Teilnahme die Historizität der geschilderten Ereignisse unterstreichen wollen. Offenbar hat sich in der Sekundärliteratur diese Sichtweise nun durchgesetzt, was Efthymiades, L'enseignement secondaire (s. Anm. 14), 260-265 erkennen läßt.

16 So Leon Diak., Hist., 5, 19-22 (Hase) [im folgenden: L.D.], auch wenn es sich dabei um einen seit Herodot (Hist. I 8, 10-11 [Stein]) fast schon inflationär gebrauchten literarischen Topos handelt; über die bereits in der Antike anzutreffende mißbräuchliche Verwendung eben dieses Topos' berichtete eindrucksvoll Luc., Hist. conscr., 305, 16-306, 2 (Macleod).

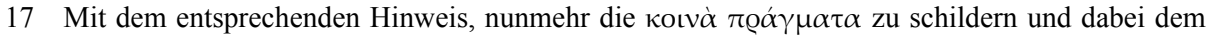
Genus der Geschichtsschreibung zu folgen, die bekanntlich das $\alpha \lambda \eta \theta$ í $\varepsilon_{\varepsilon} v$ als ihr Leitprinzip habe (dazu Arist., Rhet. 1359 b 28-32), endet die Vorrede (L.D., 5, 9-14).

18 Anaxim., Rhet. I 4, 1421b 22-32 (Chiron).

19 Arist., Rhet. I 3, 1358b 23-26 sowie I 4, 1359b 18-23 zu den Gelegenheiten, anläßlich derer die entsprechenden Reden gehalten werden sollen.

20 Gerade der letzte Begriff ist natürlich doppeldeutig: Zum einen soll die erfolgreiche öffentliche Rede das zum Ziel haben, was leicht und ohne großen Aufwand erreichbar ist (s. Anaxim., Rhet. I 4, 1421b 25 und 1422 a 15-16 [Chiron]), doch muß sie dem Zuhörer zum anderen auch eine leichte Beurteilung des geschilderten Sachverhalts erlauben (Hermog., Id. I 1, 214, 12-21 [Rabe]). Sechs kanonische „Kephalaia“ der Feldherrnrede finden sich auch bei A. Koechly (ed.), Anonymi Byzantini Rhetorica militaris nunc primum editur. Pars prior, Turici 1855, VIII 2 (= I, 7); zum @óx́ııv in der Feldherrnrede s. ebd., XII 1 (= I, 12). Das letzte Kephalaion in diesem Text behandelt das als direktes Hermogenes-Zitat (Stat. VII, 79, 7-12 [Rabe]) übernommene 
mogenes fügt diesem Kanon - je nach unmittelbarem Zweck der Reden - gele-

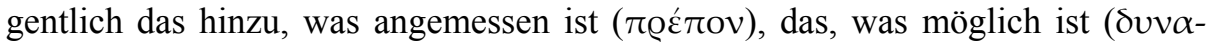

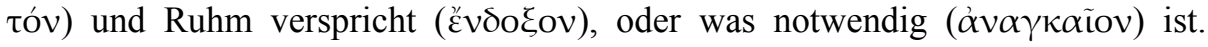
Zuletzt wäre noch eine möglichst große Verständlichkeit bzw. Klarheit des Gegenstands ( $\sigma \alpha \phi \varepsilon ́ \varsigma)$ zu nennen. ${ }^{21}$

Inwieweit man von diesem Redetypus die Feldherrnrede als ein eigenständiges Genus tatsächlich absetzen sollte, ist umstritten. Orientiert man sich jedoch am sprachlichen Vorbild bei Thukydides, scheint dies durchaus angemessen zu sein, doch bleibt immer die Abhängigkeit von der jeweiligen historischen Konstellation, die anstelle einer gewöhnlichen „Volksrede“ den logos parakletikos vor dem Heer als Vertreter dieses „Volks“ erzwingt. ${ }^{22}$ Ein solcher Rückbezug auf Thukydides läßt aber erkennen, daß es sich wie schon damals auch bei Leon Diakonos eindeutig um ein Element der literarischen Konstruktion handelt, nämlich um Reden, die im überlieferten Wortlaut mit Sicherheit niemals gehalten wurden und allein den Zweck verfolgen, das scheinbar einsame Handeln des Helden zu verdeutlichen: ${ }^{23}$ Er allein ist es, der eine bestimmte historische Situation in der rechten Weise erkennt und daher die Seinen zu einer angemessenen (Re-)Aktion

$\dot{\varepsilon} \kappa \beta \eta \sigma o ́ \mu \varepsilon v o v$, das unabhängig von Sieg oder Niederlage auf ewigen Ruhm abzielt (VIII, $3=8$ ) - ein Motiv übrigens, das sich auch für vermeintlich religiös motivierte Kriege eignet. Die Erfordernisse eines Krieges gehören dabei zweifelsohne zu den notwendigen Handlungsweisen (vgl. dazu auch D. Brodka, Geschichtsphilosophie in der spätantiken Historiographie. Studien zu Prokopios von Kaisareia, Agathias von Myrina und Theophylaktos Simokattes [Studien und Texte zur Byzantinistik 5], Frankfurt am Main u.a. 2004, 231-232).

21 Hermog., Prog. VI, 14, 6-8 und XII, 27, 1-10 (Rabe). Gerade das $\sigma \alpha \phi \varepsilon ́ \varsigma$ bzw. das stereotyp erhobene Postulat der $\sigma \alpha \phi \eta ́ v \varepsilon\llcorner\alpha$ sollte ja zu einem der wichtigsten, wenn auch für das moderne Textverständnis oft nicht mehr nachvollziehbaren Instrument der byzantinischen Stilkritik werden. Zur Thematik insgesamt sei hier verwiesen auf J. Martin, Antike Rhetorik. Technik und Methode (HdAW 2, 3), München 1974, 167-176.

22 Leimbach, Musterrhetorik (s. Anm. 2), 11-13; B. Hambsch, Feldherrnrede, in: Historisches Wörterbuch der Rhetorik 3 (1996) 225-226. Dabei ist jedoch die formale Abgrenzung zur ebenfalls situationsbedingten epideiktischen Rede zu wahren. Nur bedingt stichhaltig ist die von A. M. Taragna, Logoi historias. Discorsi e lettere nella prima storiografia retorica bizantina (Hellenica 7), Alessandria 2001, vorgenommene strikte Unterordnung des parakletikos unter den stratiotikos, die man für die spätantik-byzantinische Zeit mit Sicherheit nicht mehr durchhalten kann. Vielmehr muß man beide Genusbezeichnungen als beliebig austauschbar ansehen (s. dazu auch Taragna, ibidem, 45 mit Anm. 36 für das literarische Vorbild des Thukydides).

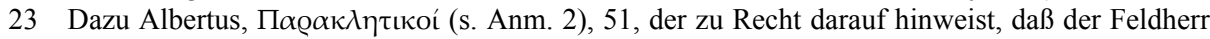
seine zuvor schon getroffene Entscheidung nicht zum Gegenstand der Rede machen darf; vgl. auch Luschnat, Feldherrnreden (s. Anm. 2), 5; Leimbach, Musterrhetorik (s. Anm. 2), 13-15 und 129. Die Historizität solcher Reden verteidigte M. H. Hansen, The Battle Exhortation in Ancient Historiography. Fact or Fiction?, in: Historia 42 (1993) 161-180, bes. 172-173 und 179-180, ohne dabei zu überzeugen, da er die von ihm als Beleg angeführten neuzeitlichen Feldherrnreden nicht vor dem Hintergrund der Antikenrezeption der Renaissance sieht und den politischen Charakter der antiken Redebeispiele völlig ignoriert. Ergänzend zu dieser Problematik auch vor dem Hintergrund der möglichen Rezeption D. A. Russell, Greek Declamation, Cambridge (u.a.) 1983, 106-117. 
führen kann. Thukydides wählte zu diesem Zweck den Typus der auch zu vergleichbaren Gelegenheiten passenden aufmunternden Rede, was wiederum Einfluß auf den übergeordneten Handlungsrahmen hat. Denn eine solche Rede hat nur Sinn, wenn das Heer als Adressat verunsichert ist oder wenn vor einer (politischen) Allgemeinheit eine Entscheidung von großer Tragweite zu rechtfertigen wäre. ${ }^{24}$ Dies jedoch hat zur Folge, daß eine solche Rede in der literarischen Komposition eines Textes einen Wendepunkt markiert, mit dem das übergeordnete Ereignis, um das es eigentlich geht, bereits im Sinne des Redners entschieden wird.

Völlig fremd war die Militärrhetorik den Byzantinern aber nicht. Darauf wies bereits Herbert Hunger hin $^{25}$ und führt als Beispiel einen anonymen Traktat zum Thema an, der womöglich aus dem 6. Jahrhundert stammt, ${ }^{26}$ also aus einer Zeit, in der die militärische Auseinandersetzung eine sehr wichtige Rolle spielte. Der Text, bei dem es sich um eine relativ freie Hermogenes-Kompilation handelt, setzt dabei für die Militärrede neben dem unvermeidlichen Prooimium sechs deklamatorische Hauptabschnitte, ${ }^{27}$ die dann der Reihe nach abgehandelt werden,

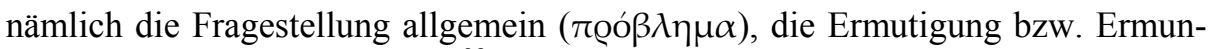
terung zum Krieg $(\pi \rho \circ \beta 0 \lambda \eta),{ }^{28}$ das ethisch-moralische Kriterium, mit dem der

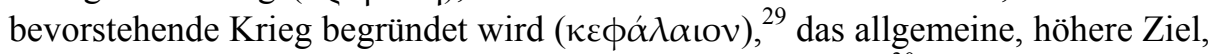

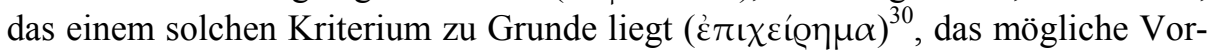
bild, das aus ähnlichen Gründen entsprechend gehandelt hat $(\dot{\varepsilon} \text { ○ } \gamma \alpha \sigma i \alpha)^{31}$ sowie die Absicht bzw. der Plan, der einem solchen Handeln zu Grunde lag ( $\dot{\varepsilon} v \theta \hat{v}-$ $\mu \eta \mu \alpha){ }^{32}$ Abschließend listet der Autor noch einige im militärischen Kontext

24 Luschnat, Feldherrnreden (s. Anm. 2), 124-125.

25 Hunger, Hochsprachliche profane Literatur (s. Anm. 5), II 328-329. Bekanntlich ließ ja auch Nikephoros Phokas eine militärtaktische Schrift verfassen, in der er seine erfolgreiche Vorgehensweise insbesondere in den Kämpfen an der Ostgrenze des Reichs darlegen ließ (G. Dagron/H. Mihăescu [éd.], Le traité sur la guérilla [De velitatione] de l'empéreur Nicéphore Phokas [963-969] [Le monde byzantin], Paris 1986); ergänzend dazu s. aber auch H. Ahrweiler, Un discours inédit de Constantin VII Porphyrogénète, in: TM 2 (1967) 393-404.

26 Koechly (ed.), Anonymi Byzantini Rhetorica militaris (s. Anm. 20). Überschrieben ist dieser

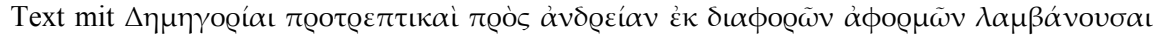
$\tau \dot{\alpha} \varsigma$ vं Textes.

27 Im wesentlichen wurden die einzelnen Begriffe sowie deren Erläuterung übernommen und aus

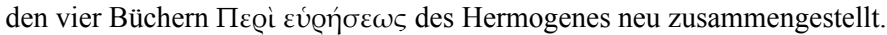

28 Beide nur sehr knapp in Koechly (ed.), Anonymi Byzantini Rhetorica militaris (s. Anm. 20), VIII, 1 (=I, 7).

29 Ebd., VIII 2-XXIV 2 (= I, 7-15). Erwartungsgemäß werden gerade die sog. Kephalaia am sorgfältigsten behandelt und mit praktischen Beispielen veranschaulicht.

30 Ebd., XXV-XXVIII (= I, 15-18).

31 Ebd., XXIX-XXXIV (= I, 18-20).

32 Im wesentlichen ebd., XXXV-XLVI (= II, 3-11), wobei jedoch hauptsächlich Redebeispiele angeführt werden. 
mögliche Redetypen auf, wobei der von Leon Diakonos verwendete logos $p a$ rakletikos (bzw. paramythetikos) natürlich nicht fehlt. ${ }^{33}$

So verwundert es kaum, daß die Geschichtsschreibung auch den großen Generälen jener Zeit die eine oder andere Rede in den Mund legte, ${ }^{34}$ die nicht nur das allgemeine, höhere Interesse des behandelten Gegenstands herausstellt, sondern den Zuhörern neben der gebührenden Ermahnung auch Mut zusprechen soll. Erfolgreich war eine solche Rede nur dann, wenn sich bei den Adressaten auch tatsächlich eine echte Ermutigung einstellte, die ein zuvor womöglich verzagtes Heer dazu befähigte, die bevorstehenden schweren Auseinandersetzungen zu den eigenen Gunsten $\mathrm{zu}$ entscheiden. Im folgenden seien also die entsprechenden Redestücke, die Leon Diakonos in seinen Text einfügte, kurz skizziert und auf ihren rhetorischen Gehalt hin untersucht.

Die erste Rede steht an entscheidender Stelle im Kontext der Rückeroberung des seit etwa dem Jahr 824 arabisch besetzten Kreta. ${ }^{35}$ Ganz dem mimetischen

33 Ebd., LVI-LVIII (= II, 16-18). Hierauf geht übrigens auch der kurze Hinweis aus Nik. II. Phokas, De Velitat. XXIII 23-29 (Dagron) zurück, der General möge gegebenenfalls eine Mut machende Rede halten. A.-M. Talbot/D. F. Sullivan (Hrsgg.), The History of Leo the Deacon. Byzantine Military Expansion in the Tenth Century (DOS 41), Washington, D.C. 2005, 73 Anm. 20 weisen zwar auf die zuletzt genannte Passage hin, doch entging ihnen leider der größere inhaltliche Kontext, in dem der gesamte Traktat steht.

34 Einen vollständigen Katalog solcher Reden aus der antiken Literatur bietet Albertus, П $\alpha \varrho \alpha-$

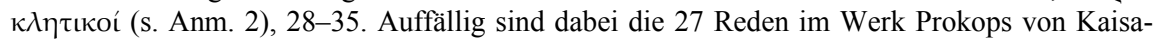
reia, der allein schon durch dieses formale Element seine enge formale Verwandtschaft mit Thukydides erkennen läßt. Allerdings schließt Albertus ihn als spätantiken Autor aus dem Kreis der näher zu untersuchenden Texte aus. Dies nachgeholt hat Taragna, Logoi historias (s. Anm. 22) (erg. auch die Rez. von M. Grünbart, in: JÖB 53 [2003] 291-292), die 221-236 für Prokop insgesamt 165 Reden auflistet, wobei sich die im Vergleich zu Albertus hohe Zahl dadurch erklärt, daß sie alle Redestücke untersucht, also auch die logoi presbeutikoi oder die symbuleutikoi (die parakletikoi s. gesondert auch bei Taragna, Logoi historias, 102-104). Wie Taragna, Logoi historias darlegt, strukturieren sie einerseits die bisweilen monotone Auflistung kriegerischer Auseinandersetzungen, andererseits geben sie dem Autor Prokop die Möglichkeit, unter dem Schutz der Prosopopoiie auch seine persönliche Meinung zu den geschilderten Dingen einfließen zu lassen. Auf diese Weise unterstützt der inflationäre Gebrauch der logoi bei Prokop in erster Linie die Lebendigkeit und den von ihm beabsichtigten Eindruck der hohen Authentizität seiner Darstellung, während die geringere Anzahl (es sind nur zehn sowie ein Gesandtschaftsbrief; s. Taragna, Logoi historias, 237-238) bzw. der gezielte Einsatz solcher Reden bei Agathias die formale Nähe des Leon Diakonos gerade zu diesem Autor zu unterstützen scheint (s. dazu auch o. Anm. 6). Dies trifft aber nur bedingt zu, da die Reden bei Agathias dem antiken Vorbild folgen und zumeist erheblich mehr Raum einnehmen als bei Leon (dazu Taragna, Logoi historias, 151-153).

35 L.D., 12, 5-13, 10. $\mathrm{Zu}$ den unterschiedlichen chronologischen Ansätzen für die sukzessive Eroberung der Insel in der byzantinischen und arabischen Geschichtsschreibung s. V. Christides, The Conquest of Crete by the Arabs (ca. 824). A Turning Point in the Struggle between Byzantium and Islam, Athen 1984, 85-95 (daraus mehr oder weniger nur übersetzt und abgeschrieben hat M. Kremp, Arabisches Kreta. Das Emirat der Andalusier [824-961], Frankfurt am Main 1995, 54-72). 
Charakter verpflichtet, den bereits Hermogenes der thukydideïschen Geschichtsschreibung zusprach, ${ }^{36}$ konstruierte Leon einen dramatischen Handlungsrahmen: Nach erfolgreicher Landung auf der Insel und einem angeblichen ersten Scharmützel, das der kaiserliche Strategos Nikephoros Phokas zu seinen Gunsten entscheiden konnte, ${ }^{37}$ war ein Spähtrupp unter Führung des als sehr zuverlässig bezeichneten Nikephoros Pastilas von den Arabern vollständig aufgerieben worden. Als Ursache für den Untergang des Pastilas und seines Trupps benennt Leon nicht etwa ein Defizit an militärischen Fähigkeiten, sondern die Mißachtung der Pflich-

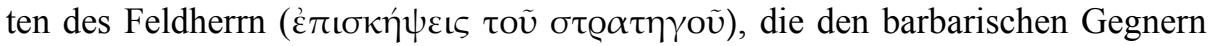
den entscheidenden Vorteil gegeben habe. Denn offenbar bot die Insel Kreta derart ungewohnte Annehmlichkeiten, daß sich die Rhomäer dem Leichtsinn und der Schwelgerei zuwandten - zwei aus der literarischen Tradition nur allzu vertrauten Gefahrenquellen. ${ }^{38}$ Als Folge kam es zu einer Entmutigung des eigenen Heeres. Außerdem sah Nikephoros die große Gefahr, daß der Gegner durch seinen Erfolg neuen $\mathrm{Mut}^{39}$ schöpfen könnte, was ihn seinerseits zu einem entschlossenen Handeln trieb: Er schnitt die arabische Hauptstadt Handaq mit einem umfangreichen Belagerungsring von ihrem Hinterland ab, um daraufhin möglichst rasch zu einer Entscheidungsschlacht zu kommen, mit der er sich ein weiteres tropaion errichten könne. Unmittelbar darauf beginnt in Gegenwart seiner Offiziere ${ }^{40}$ sein logos parakletikos im Feldherrnzelt. Diese kurze Rede ist klar strukturiert:

36 Hermog., Id., II, 411, 2-7 (Rabe). Die Tragweite der offenkundigen rhetorischen Ausgestaltung von Geschichtsschreibung in byzantinischer Zeit ist keineswegs zu unterschätzen: Zwingt sie doch dazu, einen geschilderten Handlungsablauf anhand von Parallelquellen zu überprüfen oder im anderen Fall zu einer gewissen Skepsis, was die Ereignisfolge, insbesondere aber auch die Motivation der Protagonisten betrifft. Übrigens wies bereits F. Tinnefeld, Kategorien der Kaiserkritik in der byzantinischen Historiographie von Prokop bis Niketas Choniates, München 1971, 109, darauf hin, daß man Leon Diakonos nur mit einer gewissen Vorsicht als historische Quelle auswerten könne.

37 Es ist hier nicht der Ort zu entscheiden, ob es sich bei dieser ersten Auseinandersetzung nur um eine die dramatische Konzeption des Textes unterstützende Fiktion handelt, die in den Parallelquellen fehlt. Dazu Christides, Conquest (s. Anm. 35), 177-178 (dort auch die zwei bzw. drei entsprechenden Quellenbelege).

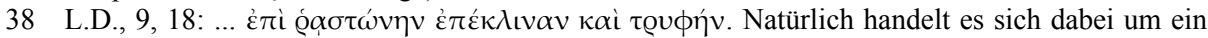
traditionelles Motiv, s. etwa Eus. Caes., Vita Const. II 14, 13 -4 (Winkelmann) oder verschiedene Zitate bei Const. Porph., De sent., u. a. 25, 10, Nr. 43 (nach Menander Prot.) (Boissevain), d.h. man sollte davon ausgehen, daß wir es mit einer idealisierten Ereignisfolge zu tun haben.

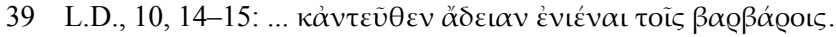

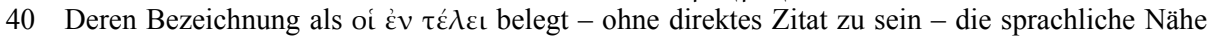
zu Thukydides, vgl. etwa Hist. IV 65, 2, 1-3 (= I, 312, 3-5 [Hude]) u.a.m., während die $\gamma \varepsilon \gamma \omega v 0 \tau \dot{\mathrm{Q}} \alpha \phi \omega v \eta$, mit der er laut Leon gesprochen habe, als mimetisch-tragisches Element anzusehen ist, das den Ernst der Situation unterstreicht. Vielleicht sollte man darin aber auch eine Regieanweisung für den öffentlichen Vortrag des Textes, etwa im Rahmen eines literarischen Theatrons, sehen. 
a) 12, 5-11: Ein gemeinsames Wissen von General und seinen Gefolgsleuten, den Ernst und die Unerträglichkeit der aktuellen Situation betreffend, wird vorausgesetzt.

b) 12, 11-16: Als notwendige Folge führt diese Situation zu einem Eingreifen der göttlichen Pronoia (für die in der hier geschilderten Konzeption auch der den General beauftragende Kaiser nur ein ausführendes Organ sein muß!), indem sie das Heer des Nikephoros Phokas für sich einsetzt.

c) 12, 16-21: Der „Sieg“ gegen die Araber gleich nach der Landung gilt als Beweis bzw. sichtbares Zeichen für das wohlwollende göttliche Handeln.

d) 12, 21-13, 3: Es schließt eine Ermahnung gegenüber möglichen Gefahren an, denn Leichtsinn und Schwelgerei, ${ }^{41}$ also der Ungehorsam gegenüber dem göttlichen Auftrag, können - wie bereits erwiesen - einen weiteren Erfolg behindern.

e) 13, 3-10: Der Schlüssel zum Erfolg ist es daher, diese Ermahnung zu beherzigen und sich auf die innere Stärke zu besinnen, die schon im eigenen

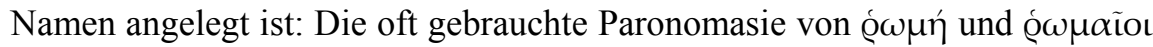
bleibt dabei jedoch unübersetzbar.

Im ersten Abschnitt werden die Rechtmäßigkeit des eigenen Handelns sowie der allgemeine Nutzen herausgestellt, dem sich der bevorstehende Krieg unterordnet, denn als die wahren Kinder Abrahams treten die Byzantiner gegen die Nachkommen der verstoßenen Sklavin des alttestamentarischen Patriarchen an. ${ }^{42}$ Der

$41 \mathrm{Zu}$ diesem Begriffspaar s. o. Anm. 38. Hierin eine direkte literarische Anspielung auf Diodorus Siculus zu sehen, wie dies Talbot/Sullivan, History (s. Anm. 33), 66, Anm. 68 tun, ist zunächst doch sehr überraschend, und sieht man den Text genauer durch, gibt es bei Leon Diakonos weitere Anlehnungen an Diodorus - wie auch an Autoren wie Dio Cassius oder Polybios, die allesamt im Rahmen der sog. Konstantinischen Exzerpte in byzantinischer Zeit neu erfaßt worden waren. Dies läßt jedoch den Rückschluß zu, daß Leon Diakonos Zugang zu diesem Material besessen habe mußte. Wahrscheinlich sollte man auch in jenem Sikeliotes Didaskalos aus dem Prooimium des Joannes Skylitzes (3, 18 [Thurn]), den Markopoulos, Byzantine History Writing (s. Anm. 3), 193 zurecht als Phantom bezeichnet, eine Verschreibung für Diodorus Siculus sehen - eben als einen jener Autoren aus dem Kreis der nicht allzu lang vorher entstandenen Exzerpten-Sammlung (zur Wertschätzung des Diodorus in byzantinischer Zeit s. auch Agathias, Hist., 64, 24-25 [Keydell]). Andere Zuweisungen bei Talbot/Sullivan wie etwa die der engli-

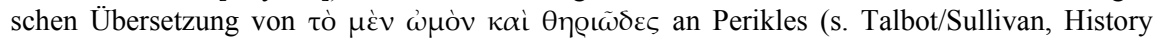
[s. Anm. 33] 65, Anm. 62) lassen jedoch einen allzu unkritischen Umgang mit dem elektronischen TLG erkennen, da den Bearbeitern offenbar des Empfinden für den rhetorisch-topischen Gebrauch solcher Wendungen fehlt, wie sie insbesondere auch bei den Kirchenvätern häufig begegnen; zu dieser Problematik allgemein s. jetzt D. R. Reinsch, Zum Edieren von Texten: Über Zitate, in: E. Jeffreys (Hrsg.), Proceedings of the 21st International Congress of Byzantine Studies. London, 21-26 August 2006. I: Plenary Papers. Aldershot 2006, 299-309.

42 Möglicherweise flocht Leon Diakonos auf diese Weise die bekannte Nikephoros-Akklamation $\kappa \alpha \lambda \tilde{\omega} \varsigma \tilde{\eta} \lambda \theta \varepsilon v \dot{\eta} \alpha \dot{\alpha} v \alpha i ́ \varrho \varepsilon \sigma \iota \varsigma \tau \tilde{\omega} \nu \tau \tilde{\eta} \varsigma$ 'A $\gamma \alpha \varrho$ nach Const. Porph., De cer., I 5 (51, 12-13 Reiske) in seinen Text ein; s. dazu O. Kresten, Pallida mors Saracenorum. Zur Wanderung eines literari- 
Krieg ist demnach eine gerechte Sache und nützt den Byzantinern ohnehin, da Kreta als militärische Basis für Raubzüge gegen das Reich diente, was historisch sicherlich auch als das wichtigste Motiv für die angestrebte Rückeroberung der Insel anzusehen ist. Allerdings duldet Gott dieses offenkundige Unrecht nicht länger und entsendet Nikephoros Phokas, was den Krieg als notwendige Folge des göttlichen Eingreifens qualifiziert, den Menschen nicht verhindern dürfen. Als Beweis dafür, daß man eine gute Sache verfolgt, dient der (angebliche) Sieg bei Landung der Truppen. Den Erfolg behindern kann höchstens der menschliche Ungehorsam bzw. die Überheblichkeit, wofür - wenn auch ohne Verantwortung des Generals, der es besser gewußt hätte - der Erweis bereits erbracht worden war. Man muß nur dessen Ratschläge beherzigen, und schon wird ein Sieg leicht möglich sein. Es läßt sich also klar belegen, daß Leon Diakonos die kurze Rede des Nikephoros Phokas um einige der vom literarischen Genos geforderten Leitsätze herum konstruierte.

Die Rede erreicht offenbar ihr Ziel, denn gemäß dem übergeordneten Zweck des logos parakletikos ermuntert sie das gesamte Heer, ${ }^{43}$ wenngleich dies im Widerspruch zu der Eingangsszene steht, da ja nur die Offiziere Adressaten dieser Rede waren. ${ }^{44}$ Zentraler Ausdruck an dieser Stelle ist aber „das Heer faßte wieder

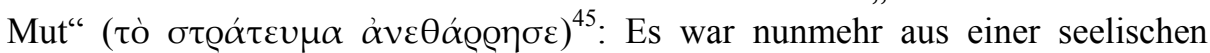
Regung heraus ${ }^{46}$ dazu bereit, seinem Anführer in allen Dingen Folge zu leisten. ${ }^{47}$ Der philosophische Kontext, im dem diese Formulierung steht, wird auch durch den Wortgebrauch von $\dot{\alpha} \tau \varrho \varepsilon \mu \varepsilon \tilde{\imath} v$ und $\eta_{\varrho} \varepsilon \mu \varepsilon \tilde{\imath} v$ belegt, ${ }^{48}$ das Nikephoros seinen

schen Topos von Liudprand von Cremona bis Otto von Freising und zu seiner byzantinischen Vorlage, in: RHM 17 (1975) 32 und 49-55.

43 Zum musterhaften paränetisch-anspornenden Charakter solcher Reden, die gleichzeitig die überragende historische Rolle des Feldherrn veranschaulichen sollen, s. Luschnat, Feldherrnreden (s. Anm. 2), 113-114, sowie Leimbach, Musterrhetorik (s. Anm. 2), 13-15.

44 Maur., Strat., VII A 4 (Dennis) wies dazu an, daß Anfeuerungsreden nicht vor dem ganzen Heer, sondern jeweils vor den einzelnen Truppeneinheiten zu halten sein. Allerdings wird dort nicht nur das Offizierskorps als Adressat bezeichnet.

45 L.D., 13, 11.

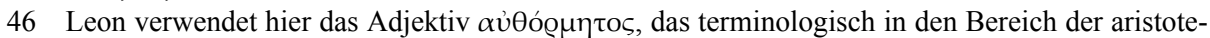
lischen Seelenlehre gehört, vgl. Simpl., Comm. in de An. 406 b 7 = CAG, XI, 37, 28-30 (Hayduck). Gerade auf diese Weise wird der Erfolg der Rede ausgedrückt, die die Seele der Zuhörerschaft in Bewegung versetzt und ohne willentliches Zutun des einzelnen den weiteren Verlauf der Ereignisse evoziert. Die im LBG s.v. (= S. 230) angegebene Bedeutung ist unzutreffend, da nicht als terminus technicus erkannt.

47 Als Beleg für die literarisch-rhetorische Ausgestaltung auch dieser Abschlußszene sollte man den homerischen Sprachgebrauch (cf. Il., 3, 272; 19, 259 u.a.m.) erachten. Das bei Homer mit Atreus verbundene Epitheton steht hier demnach - wie auch bei anderen byzantinischen Historikern, z. B. Michael Psellos - für die gemeinschaftliche, Furchtlosigkeit demonstrierende Präsentation der gezogenen Schwerter.

48 Dazu Arist., De an. 406 a 23-27, besonders aber auch in seinem Kapitel über die göttliche Pronoia Plotin, En., III 2, 4, 13-16 (edd. Henry/Schwyzer). 
Soldaten auferlegt, bis er selbst als Beauftragter der göttlichen Vorsehung das weitere Vorgehen veranlassen würde. Es versteht sich von selbst, daß der General nach dieser Rede einen glänzenden Sieg feierte, indem er ein zur Befreiung von Handaq aufgestelltes arabisches Entsatzheer in einem Überraschungsangriff besiegte und sich nunmehr auf die Belagerung der Stadt sowie deren endgültige Eroberung konzentrieren konnte. ${ }^{49}$

Die zweite Rede ${ }^{50}$ soll Leon Phokas gehalten haben, der Bruder des Nikephoros, den Kaiser Romanos II. mit der Befriedung des südlichen Kleinasien beauftragt hatte. ${ }^{51}$ Im Gegensatz zur ersten Rede, die zwar im größeren Kontext des kaiserlichen Befehls stand, dabei aber das Walten der göttlichen Pronoia als movens in den Mittelpunkt stellte, wird dieser Auftrag expressis verbis zum Ausdruck gebracht. Leon befand sich in einer für ihn und sein Heer scheinbar aussichtslosen Lage, da ihn eben jene kaiserliche Order mit einer feindlichen Übermacht konfrontierte, die er in offener Feldschlacht nicht würde bezwingen können. ${ }^{52}$ In dieses sichtbare, vom Kaiser selbst verursachte Dilemma hinein

49 Zur arabischen Reaktion auf die Belagerung von Chandax sowie zur Chronologie der Ereignisse im einzelnen s. F. Dölger, Regesten der Kaiserurkunden des Oströmischen Reiches, Regesten von $867-1025$, neu bearb. von A. E. Müller unter verantwortl. Mitarb. v. A. Beihammer, München 2003, I, 1, 2, Nr. 687e (mit Literatur).

50 L.D., 20, 13-21, 13

$51 \mathrm{Zu}$ den hier beschriebenen historischen Vorgängen sowie zu Leon Phokas s. J.-Cl. Cheynet, Les Phocas, in: G. Dagron/H. Mihăescu (Hrsgg.), Le traité sur la guérilla (s. Anm. 25), 301-306 (dort auch weitere Literatur) sowie J. Haldon, The Byzantine Wars. Battles and Campaigns of the Byzantine Era, Stroud 2001, 91-94; zur seiner Bewertung bei Leon Diakonos s. A. Markopoulos, Gender Issues in Leon the Deacon, in: ders., History and Literature of Byzantium in the

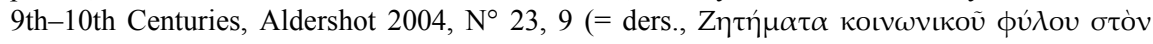

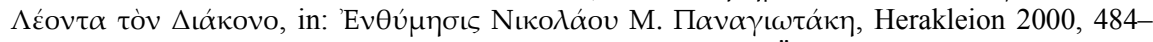
485); erg. dazu aber Tinnefeld, Kaiserkritik (s. Anm. 36), 116. Übrigens ignoriert Leon Diakonus den gesamten Komplex der vorangegangenen diplomatischen Kontakte der Byzantiner sowohl mit den Hamdāniden, als auch mit den Tarsenern, von denen im folgenden noch die Rede sein wird; siehe dazu M. Featherstone, The Great Palace as Reflected in the De Cerimoniis, in: F. A. Bauer (Hrsg.), Visualisierung von Herrschaft. Frühmittelalterliche Residenzen. Gestalt und Zeremoniell (BYZAS 5), Istanbul 2006, 56 (mit Stellenangaben), sowie zu den Tarsenern F. A. Bauer, Potentieller Besitz. Geschenke im Rahmen des byzantinischen Kaiserzeremoniells, in: ebd., 154-162.

52 L.D., 19, 12-20, 7. Tatsächlich handelt es sich um eine immanente Kaiserkritik, da die unmittelbare Umsetzung des kaiserlichen Auftrags den General und sein Heer unweigerlich in den Tod geführt hätte. Zur Kritik an Romanos II. bei Leon Diakonos s. Tinnefeld, Kaiserkritik (s. Anm. 36), 109-110. In diesem Zusammenhang sei ein weiteres aufschlußreiches Detail angemerkt, das auch als Kaiserkritik gelten muß: Leon Diakonos erwähnt 6, 15-7, 8 nur knapp die im Jahr 949 mit einem immensen militärischen Aufwand (s. Const. Porph., De Cer., II 45 [664-678 Reiske]) durchgeführte Unternehmung des Patrikios Konstantinos Gongyles gegen das arabische Kreta. Sein literarisches Konstrukt geht nun davon aus, daß der Kaiser die Hybris der Kreter nicht länger ertragen habe $(6,16-17)$, währende es im Falle von Nikephoros Phokas die göttliche Pronoia gewesen war, die das allgemeine Übel nicht länger habe dulden können $(12,11$ 13). Der Mangel des göttlichen Auftrags hätte - wie hier im Falle des Leon Phokas - gegebe- 
plazierte Leon Diakonos seinen zweiten logos parakletikos, durch dessen Befolgung nunmehr die für die Byzantiner ungünstige Lage sukzessive umgekehrt werden konnte. Leon Diakonos sagt sogar dreimal, daß es sich dabei in formaler Hinsicht um eine Paränese ${ }^{53}$ handele, die folgerichtig den Zweck haben sollte, das Heer „mit Worten zuzurüsten“ und seinen Mut zu steigern, sobald es tatsächlich zu militärischen Auseinandersetzungen käme. ${ }^{54}$ Während aber bei Nikephoros das Feldherrnzelt Ort der Rede war, spricht sein Bruder zum ganzen Heer, das nach dem Zeugnis des Leon Diakonos zumindest doch überschaubar gewesen sein muß. ${ }^{55}$ Auch diese Rede, die eindeutige Anspielungen an Prokop von Kaisareia erkennen läßt, ${ }^{56}$ ist klar strukturiert:

a) 20, 13-18: Leon legt die Gegebenheiten dar, und zwar unter Anspielung auf das kaiserliche Wissen um die Befähigung jedes einzelnen Soldaten. Dramatisch überhöht wird die Szene durch die Personifikation Kleinasiens, das massiv unter den Raubzügen des Hamdāniden Ali Sa'if-ad Daulah ${ }^{57}$ litt.

b) 20, 18-24: Ermahnung - wieder unter Anspielung auf den Mut der Soldaten -, der Taktik des Generals Folge zu leisten. Den logos parakletikos verraten dabei die Worte $\pi \alpha \varrho \alpha i ́ v \varepsilon ı v$ und $\sigma v \mu \beta o u \lambda \varepsilon v \varepsilon \varepsilon v$ (p. 20, 18), denn nur wer aufs beste beraten ist, wird - wie auch in den vorangegangenen Kämpfen ${ }^{58}$ seinen Feind besiegen. ${ }^{59}$

nenfalls durch strategische Fähigkeiten wettgemacht werden können. Dies traf aber für die Unternehmung des Jahres 949 nicht zu, da Konstantinos Gongyles der Situation aufgrund seiner Lebensumstände nicht gewachsen war (7, 3-7).

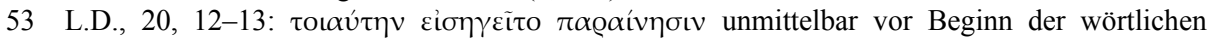
Rede; außerdem 20, 10 und 21, 24.

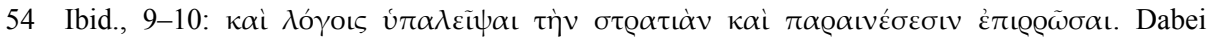
scheint das $\lambda$ ó $\gamma$ oı $v ं \pi \alpha \lambda \varepsilon \tilde{\psi} \psi \alpha \iota$ zumindest verdächtig, d.h. der Text sollte hier in der bislang vorliegenden Form nicht als Argumentationsbasis dienen. Gleichwohl könnte es sich hier um eine sprachliche Reminiszenz an Rom. Mel., Cant. 40, 1, 4-5 (Maas/Trypanis) handeln, also an die vorösterliche Salbung des scheinbar unterlegenen, in Wahrheit aber doch siegreichen Leib Christi.

55 L.D., 19, 23-20, 1.

56 Die entsprechenden Belege s. jetzt bei Talbot/Sullivan, History (s. Anm. 33), 73-74 mit Anm. 21-24, wobei jedoch im Rahmen einer kommentierten Übersetzung unbedingt nachzuprüfen gewesen wäre, welche Texte Prokop seinerseits zitierte!

$57 \mathrm{Zu}$ den arabischen Erfolgen in der Mitte des 10. Jahrhunderts s. E. Honigmann, Die Ostgrenze des byzantinischen Reiches von 363 bis 1071 nach griechischen, arabischen, syrischen und armenischen Quellen (= A. A. Vasiliev, Byzance et les Arabes III), Brüssel 1935, 80-82. Insgesamt schönt Leon Diakonos die Dinge sicherlich zu Gunsten der Byzantiner und unterscheidet auch nicht zwischen einzelnen arabischen Gruppen und Stämmen, was jedoch der byzantinischen literarischen Praxis entspricht.

58 Es liegt hier eine teils wörtliche Anspielung an Eus. Caes., Vita Const., I 6 (Winkelmann) vor, die auch später noch bei anderen historiographischen Autoren des 11. Jahrhunderts begegnen sollte.

59 Es verwundert nicht, daß diese paränetische Formel in Verbindung mit der Aufforderung, einen bestimmten Sachverhalt einzusehen und umzusetzen, sehr häufig in den Predigten des Joannes 
c) 20, 24-21, 11: Replik des bereits Gesagten. Die Gegebenheiten erlauben keine offene Feldschlacht, wobei durch die ausführlichere Schilderung eine rhetorische Klimax erzielt wird. Das kampfesfreudige byzantinische Heer muß nunmehr durch Worte gebunden werden, da ein ungezäumtes, tollkühnes Handeln $^{60}$ aufgrund der zahlenmäßig drückenden Überlegenheit der Gegner ${ }^{61}$ den Untergang brächte, während ein rationales Planen Rettung verheißt. ${ }^{62}$

d) 21, 12-23: Leon Phokas enthüllt seine Taktik, die drin besteht, einen zermürbenden Guerillakrieg ${ }^{63}$ gegen die hạamdānidischen Verbände zu führen. Ein Erfolg wird eintreten, wenn die Soldaten nicht nach eigenem Gutdünken handeln, sondern die Initiative dem General überlassen, der sie im geeigneten Moment zum Kampf auffordern wird. ${ }^{64}$

Zunächst argumentiert Leon Phokas mit dem, was möglich ist: Hätte der Kaiser nicht die Überzeugung gehabt, daß ein Sieg erreicht werden könne, wäre das gesamte Unternehmen wohl kaum zustande gekommen. Außerdem verfolgt man ja eine gerechte Sache, da Kleinasien sehr unter den gegebenen Umständen leidet. Dennoch wird ein Sieg nur unter bestimmten Umständen zu erreichen sein, die notwendigerweise den absoluten Gehorsam gegenüber dem General verlangen, der allein die geeigneten, dem Nutzen aller dienenden Ratschläge erteilen kann. Die Wiederholung verstärkt das bereits Gesagte ein weiteres Mal, denn das Ziel ist ein Gutes, aber um es zu erreichen, gilt es die Wahl der geeigneten bzw. an-

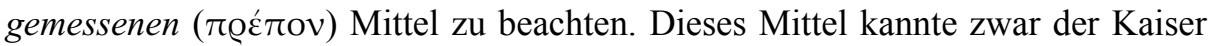
nicht, dafür jedoch Leon Phokas, der einen Sieg auf der Grundlage von rationalem Handeln für möglich hält, das darin besteht, die offene Feldschlacht zu meiden. Vor allem dürfen die Soldaten nicht aus eigenem Antrieb handeln, sondern müssen ihre individuelle Tapferkeit dem allgemeinen Nutzen unterordnen, der im Gehorsam gegenüber dem General und seinen taktischen Empfehlungen liegt.

Chrysostomos begegnet (z.B. Epist. X ad Olymp., 1d = 154, 16-22 [Malingrey]; Hom. XLIV in I Epist. ad Cor., PG 61, 380, 31-32 u.a.m.).

60 Cf. Const. Porph., De sent., 110, 27-30 (Boissevain). Dieses Argument findet sich natürlich bereits in der antiken Geschichtsschreibung sehr häufig; s. außerdem Dio Cassius, Hist., I, 90, 4-9 (Fragm. Zon.; Boissevain), der die bereits in der Jugendzeit geübte Kampfbereitschaft als römische Tugend nennt.

61 Womöglich spielt der Text hier an Hom., Il. 16, 76-79 an, zumal auch diese Formulierung in späteren Texten noch belegt ist.

62 Wiederum liegt ein literarischer Topos vor, etwa Demosth., Or. XXV in Aristog., 32, 8-33, 1 (Butcher) od. Polyb., Hist., III 63, 10-12 = 289, 6-11 (Büttner-Wobst).

63 Bes. L.D., 21, 14-16. Erinnert sei in diesem Zusammenhang nochmals an die von Nikephoros II. Phokas in Auftrag gegebene Abhandlung über den Guerillakrieg (s. o. Anm. 25). Auffällig ist aber auch die inhaltliche Parallele zu Const. Porph., De sent., 234, 14-27 (Boissevain)!

64 Die Szenerie stimmt übrigens teils wörtlich überein mit Xenoph., Anab. III 4, 4-5 (Hude), was gegen eine mögliche Historizität dieser Rede spricht. Zur Forderung des absoluten Gehorsams vgl. auch oben Anm. 46 und 47. Zur Überlegenheit des Feldherrn vgl. Brodka, Geschichtsphilosophie (s. Anm. 20), 112-113 und 115-118 (zu Belisar). 
Diese Rede hatte - wie nicht anders zu erwarten war - den beabsichtigten Erfolg und wird von den Zuhörern mit lautem Beifall quittiert, ${ }^{65}$ wobei Leon Phokas als bester Ratgeber des Heers akzeptiert wird, ${ }^{66}$ dem es in Zukunft folgen will. Wie schon zuvor bei Nikephoros zeigte für unseren Autor auch das Vorgehen Leons großen Erfolg: Die Hamdāniden werden besiegt, und der General selbst also nicht etwa der Kaiser! - ist es, der Kleinasien rettet. ${ }^{67}$ Einzelheiten über die Kämpfe erfährt man von Leon Diakonos relativ wenige, ${ }^{68}$ aber diese spielen für ihn ohnehin keine Rolle. Vielmehr dominiert die Rede das ganze Geschehen, und inhaltlich gelingt unserem Historiker auf diese Weise das kleine Kunststück, daß Kaiser Romanos II. als Auftraggeber völlig im Hintergrund bleibt. Die konkrete Handlungsinitiative und vor allem die Auflösung der Aporie, wie ein zumindest nach Auskunft des Textes zahlenmäßig weit überlegener Gegner zu bezwingen sei, war allein Leistung des Leon Phokas. Nach byzantinisch-römischem Selbstverständnis wäre dies eigentlich Aufgabe des Kaisers selbst gewesen, ${ }^{69}$ doch Heer und Volk wissen, wem sie ihre Rettung und ihren Erfolg in Wahrheit zu verdan-

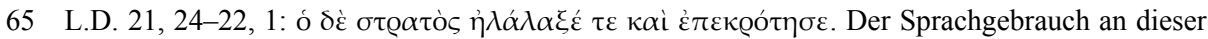
Stelle ist ein apokalyptischer und Jo. Dam., Laud. in s. Barb. mart., in: PG 96, A13-B8 (CPG

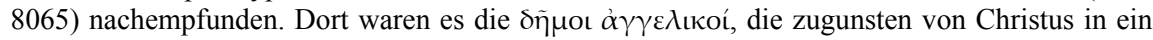
Kampfgeschrei ausbrachen und ihm applaudierten, um dann gemeinsam gegen die finsteren Dämonen auszurücken.

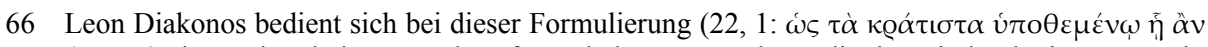

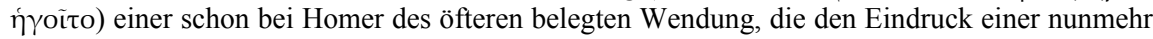
verschworenen Kampfesgemeinschaft stärken soll - aber als sprachlicher Antiquismus als ein Indikator für die Fiktivität der Szene gewertet werden kann.

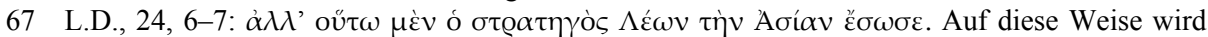
die Szene, die zu Beginn der Rede die Klage Kleinasiens anführte (L.D., 20, 15), formal und sprachlich abgeschlossen. Der größere Kontext kann sich nunmehr dem eigentlichen Helden der Erzählung, nämlich Nikephoros Phokas, widmen.

68 Die Ereignisse ausführlich dargestellt hat M. Canard, Histoire de la Dynastie des H'amdanides de Jazîra et de Syrie (Publ. de la Faculté des Lettres d'Alger II, 21), Paris 1953, 755-827, der auch die durch Leon Diakonos vermittelte klare byzantinische Überlegenheit relativiert; s. außserdem E. McGeer, Sowing with the Dragon's Teeth: Byzantine Warfare in the Tenth Century (DOS 33). Washington, D.C., 1995, 225-248.

69 Literarisches Vorbild ist hier womöglich der Triumph, den Kaiser Justinian I. dem über die Vandalen siegreichen Belisar im Jahr 534 zugestand (s. Prok. Caes., De bell., IV 9, bes. 1-5 [Veh], und M. McCormick, Eternal Victory. Triumphal Rulership in Late Antiquity, Byzantium, and the Early Medieval West, Cambridge u.a. 1986, 167-168) - was seinerzeit nach Prokop das kaiserliche Mißtrauen gegenüber seinem General verstärkte; s. dazu auch W. Treadgold, A History of the Byzantine State and Society, Stanford 1997, 185 und 495. Treadgold wies bereits darauf hin, daß der Triumph, den wenig später Nikephoros Phokas in Konstantinopel feiern durfte (L.D., 28-29), ungleich prächtiger ausfiel als der seines Bruders Leon. Möglicherweise sollte man dabei aber an eine feine sprachliche Abstufung denken, die Leon Diakonos hier mit Blick auf Nikephoros als den späteren Kaiser in seinen Text einfügte. 
ken haben - weswegen Leon nach seiner Rückkehr einen Triumphzug im Hippodrom der Hauptstadt abhalten darf. ${ }^{70}$

Auch die dritte Rede ${ }^{71}$ steht bei Leon Diakonos an exponierter Stelle: Nach dem Tod des Kaisers Romanos II. befindet sich das Reich in Unruhe, da die Frage der Nachfolge auf dem Kaiserthron nicht geklärt ist. Die Rolle des „Schurken im Hintergrund" übernimmt in der Szenerie des Leon der Parakoimomenos und Eunuch Joseph Bringas, der für sich das unabwendbare Verderben in der Person des Nikephoros Phokas aufziehen sieht, freilich ohne selbst etwas gegen ihn zu vermögen. Einer seiner Helfershelfer, der später an den Folgen eines gezielten Wurfs mit einem Tontopf ${ }^{72}$ verstorbene Patrikios Marianos, rät Bringas zu einer Intrige: Er solle sich mit Joannes Tzimiskes, dem obersten General des Nikephoros verbünden, der als einziger dazu in der Lage sei, diesen Widersacher für ihn aus dem Weg zu räumen. ${ }^{73}$ Doch Joannes bleibt loyal und verrät den geplanten Anschlag

70 L.D., 24, 3-6; M. McCormick, Triumph, -zug, in: LexMA 8, Sp. 1025, weist wohl darauf hin, $\mathrm{da} \beta \mathrm{ab}$ dem 8 . Jahrhundert auch die siegreichen Generäle als Triumphatoren auftreten durften, was aber umgekehrt als ein Zeichen der Schwäche des Kaisertums zu werten sein könnte, wie es die byzantinischen Geschichtsschreiber mit Blick auf die römischen Imperatoren wahrnahmen. Allerdings bringt Leon Diakonos $(23,19-20)$ auch klar zum Ausdruck, daß der triumphale Einzug des Generals mit Erlaubnis des Kaisers erfolgte, d.h. der Verdacht einer möglichen Usurpation wird von vornherein ausgeräumt; erg. auch M. McCormick, Eternal Victory (s. Anm. 69), 137-178. Positiv könnte man daraus den Schluß ziehen, daß sich spätestens mit Justinian I. das Selbstverständnis des Kaisertums geändert hatte: Denn nicht mehr der Herrscher selbst muß notwendigerweise in den Krieg ziehen, sondern kann diese Aufgabe - nicht zuletzt zum Schutz der eigenen Person - delegieren, auch wenn dies der eine oder andere Historiker als unangemessen empfunden haben mag. Die Komnenenkaiser folgten aber wieder dem Idealtyp des kaiserlichen Feldherren, woüber zu Beginn des 14. Jhs. etwa Nikephoros Blemmydes in seinem „Basi-

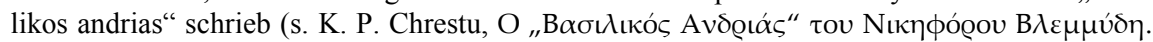

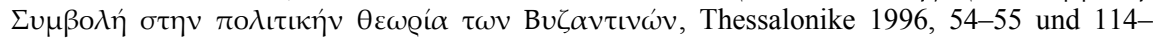
119).

71 L.D., 42, 1-43, 18. Vor dem Gebrauch der Übersetzung dieser Passage bei F. Loretto, Nikephoros Phokas, der bleiche Tod der Sarazenen, und Johannes Tzimiskes. Die Zeit von 959 bis 976 (Byzantinische Geschichtsschreiber 10), Graz/Wien/Köln 1961, 45-47, sei gewarnt.

72 L.D., 46, 13-14. Hier erkennt man deutlich ein komisches Element, das auch Theoph. Cont. berichtet (s. Talbot/Sullivan, History [s. Anm. 33], 96 Anm. 55). Unabhängig vom Gegenstand, der Marianos tatsächlich traf, liegt wohl eine literarische Anspielung auf den geisteskranken, weil von einem Dachziegel am Kopf getroffenen römischen Legaten und Ratgeber Mancinus vor (Polyb., Hist. XXXVI 14, 2 [ed. Büttner-Wobst] = Diod. Sic., Bibl. hist., XXXII, 20 [edd. Dindorf/Fischer]): Auch Marianus trat zuvor als Ratgeber auf! Gegner dieser altrömischen legatio war vor allem Scipio Africanus, den die Phokaden zu ihrem Stammvater erklärt hatten (dazu G. Weiß, Antike und Byzanz. Die Kontinuität der Gesellschaftsstruktur, in: HZ 224 [1977] 545). Da diese Passage auch in die Konstantinischen Exzerpte wanderte, liegt wieder ein Argument dafür vor, daß Leon Diakonos Zugang zu diesen Texten besaß (s. o. Anm. 41). Einiges spricht sogar dafür, daß Leon an der Erstellung von De sententiis beteiligt gewesen sein könnte, doch kann dies an dieser Stelle nicht näher erörtert werden.

73 Vgl. J. Haldon, Warfare, State and Society in the Byzantine World, 565-1204, London 1999, $230-231$. 
auf seinen Blutsverwandten, was nun Nikephoros - möchten er selbst und seine Familienangehörigen am Leben bleiben - zu einem weiteren Feldzug zwingt. ${ }^{74}$ Dieser richtet sich nun nicht gegen einen äußeren Feind, sondern gegen die byzantinische Hauptstadt - und war inzwischen unvermeidlich geworden, da das Heer seinen Generalissimus auf Betreiben des Joannes Tzimiskes zum Kaiser ausgerufen hatte. Wie es Leser und Hörer eines solchen Textes voraussetzen, zögerte Nikephoros Phokas zunächst, ${ }^{75}$ aber zuletzt beugt er sich den Erwartungen seines Heeres und zieht sich als sichtbares Zeichen der Herrschaft die purpurfarbene kaiserlichen Stiefel an, die sich nach Leons Darstellung bereits im Marschgepäck des künftigen Herrschers befunden haben müssen. Jedenfalls erfordert es die dramatische Stellung des nun folgenden logos parakletikos, daß er für sein Heer eindeutig als Kaiser zu erkennen gewesen sein muß, wofür sich die roten Stiefel besonders angeboten hätten. ${ }^{76}$ Folgerichtig setzt die Rede unter Anspielung auf die sichtbaren Herrschaftszeichen ${ }^{77}$ ein:

a) 42, 1-7: Nikephoros stellt sich unfreiwillig der Aufgabe, die an ihn herangetragen wurde, nämlich die Kaiserwürde anzunehmen, weist aber bereits vorsorglich den möglichen Vorwurf der Usurpation ${ }^{78}$ zurück. Allerdings sieht

74 L.D., 39, 14- 41, 21, bes. 42, 6, wo er explizit die eigene Sicherheit nennt. Zur Beurteilung der Kontroverse s. auch H.-G. Beck, Senat und Volk von Konstantinopel. Probleme der byzantinischen Verfassungsgeschichte (Bayerische Akademie der Wissenschaften, Sb. Phil.-hist. Kl. 1966/6), München 1966, 61 (= ders., Ideen und Realitäten in Byzanz. Gesammelte Aufsätze, London 1972, Nr. 12).

75 G. Dagron, Empereur et prêtre. Étude sur le „césaropapisme“ byzantin, Paris 1996, 94-95, nennt das politische Kalkül, das Nikephoros zunächst noch habe zögern lassen, im vollen kaiserlichen Ornat zu erscheinen.

76 Ausführlich wies bereits O. Treitinger, Die oströmische Kaiser- und Reichsidee nach ihrer Gestaltung im höfischen Zeremoniell. Vom oströmischen Staats- und Reichsgedanken, Darmstadt ${ }^{2} 1956,25$ Anm. 74, auf diese Stelle hin, die vom historischen Tatbestand nur bedingt plausibel erscheint. Literarisch hat Leon Diakonos die Szene aber sehr geschickt gestaltet, denn der purpurne Feldherrnmantel wäre die von einem größeren Heer sicherlich besser wahrzunehmende Kaiserinsignie gewesen, die ihn zudem auch als obersten Feldherrn ausgezeichnet hätte (vgl. Treitinger, 24-25). Somit kann er aber noch auf die Anerkennung durch Volk und Senat der Hauptstadt warten, bevor er endgültig in den kaiserlichen Ornat gekleidet wird. Die Legitimität dieses Handelns betont dagegen der insgesamt weit realistischere Parallelbericht bei Const. Porph., De cer., I 96 (434, 13-15 Reiske).

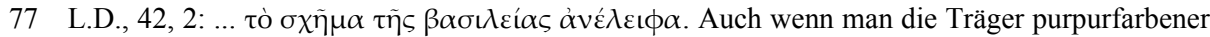
Schuhe in Byzanz als verdächtig empfand (Beispiele dazu bei H. Hunger, Reich der neuen Mitte. Der christliche Geist der byzantinischen Kultur, Graz/Wien/Köln 1965, 85-86), so sind Purpurmantel und Diadem entgegen der Aussage Leons doch die eindeutigeren Herrschaftszeichen, da sich Stiefel gegebenenfalls noch verbergen ließen!

78 Nikephoros vertraut also nicht nur auf eine Ausrufung durch das Heer, sondern will abwarten, wie er in Konstantinopel aufgenommen wird. Damit verhält er sich so wie etwaige Thronprätendenten aus den beiden vorangegangenen Jahrhunderten (dazu M. J. Leska, Legalization of Usurpers' Power in Byzantium from the Seventh to the First Half of the Ninth Century, in: W. Ceran [Hrsg.], Historija Bizancijum [Acta Universitatis Lodziensis. Folia Historica, 56], Łódź 1996, 
er - neben seiner eigenen Sicherheit - die des gesamten Staatswesens gefährdet, was ihn zu einem Kampf ${ }^{79}$ für das Allgemeinwohl ${ }^{80}$ zwingt.

b) 42, 7-10: Zur Bekräftigung bezeugt Nikephoros vor der göttlichen Vorsehung, daß er unter Einsatz seines Lebens bereit sei, diese Aufgabe zu übernehmen.

c) 42, 10-21: Der Anlaß für sein Handeln sind die „widersinnigen“ Ränke des Eunuchen Joseph Bringas, die das Heer des Nikephoros nicht duldet. Als Gegenleistung verspricht er seinerseits ohne Wanken mit göttlichem Beistand zu herrschen. ${ }^{81}$ Davor liegt aber noch eine heftige Auseinandersetzung, die die Soldaten nur mit einer der Größe der Aufgabe angemessenen Gesinnung bestehen werden. ${ }^{82}$ Denn der Neid auf den zukünftigen Träger der Kaiserwürde werde auch die Härte der Auseinandersetzung bestimmen.

d) 42, 21-43, 8: Nicht Barbaren gilt es nunmehr niederzuringen, sondern den politisch fehlgeleiteten römischen Staat, respektive dessen gut gesicherte Hauptstadt, was nur mit der richtigen inneren Einstellung zu bewerkstelligen sei. $^{83}$

e) 43, 8-15: Versicherung des göttlichen Beistands, da die Gegner die zuvor getroffenen Vereinbarungen brachen. Damit wird auch die angestrebte „Einnahme" des Staates legitimiert, die - wie eingangs erwähnt - im Falle des Scheiterns als Usurpation gelten würde. ${ }^{84}$

170-171 und 174-175). Const. Porph., De cer. I 96 (496,14-497,3 Reiske) besagt jedoch, daß sich Nikephoros Phokas ganz bewußt erst in Konstantinopel mit dem vollen kaiserlichen Ornat einkleiden lassen wollte. Die Szenerie bei Leon Diakonos soll also nur die dramatische Spannung erhöhen.

79 Leon Diakonos verwendet hier den Begriff des $\alpha \gamma \omega \dot{\omega} v(42,6)$, wobei es sich um einen neutestamentarischen Sprachgebrauch handelt, vgl. etwa Phil 1, 29-30, Kol 2, 1 u.a.m. Man kann jedoch auch an einen rhetorischen Agon denken, da der neue Kaiser die Bevölkerung Konstantinopels erst noch für sich gewinnen muß - was man durchaus als ein demokratisches Element zu bewerten hätte; daß dieser Sprachgebrauch in der byzantinischen Historiographie nicht fremd war, zeigt etwa auch der logos symbuleutikos des kolchischen Königs Aietas bei Agathias, der

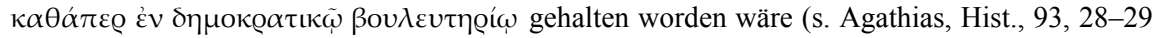
[Keydell]) bzw. Taragna, Logoi historias (s. Anm. 22), 158.

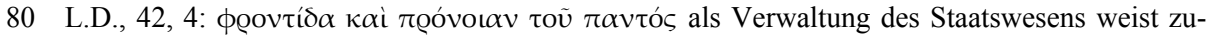
rück auf Eus. Caes., De laud. Const., XII 7,2-6 (Heikel), wo ausgehend vom göttlichen Vorbild eine ganze Reihe der herrscherlichen Tugenden und Pflichten aufgezählt sind.

81 Einige der hier für die Gegner verwendeten Begriffe sind 2 Petr 2, 8-11 entlehnt, was die Rede wiederum in einen apokalyptischen Rahmen stellt.

82 Nikephoros geht hier terminologisch genauso vor wie in seiner ersten Rede (s. o. Anm. 41).

83 Mit dem spätantiken Begriff des $\gamma \varepsilon v v \alpha$ ĩov $\pi \alpha \varrho \alpha ́ \sigma \tau \eta \mu \alpha$, der wiederholt auch über die Konstantinischen Exzerpte überliefert wurde (in unserem Zusammenhang läge De sent. 399, 19, Nr. 455 [Boissevain] bes. nahe), ist immer ein Handeln aus ganz persönlichem Antrieb heraus gemeint.

84 Die Rede beginnt L.D., 42, 1 expressis verbis mit der Abwehr der Vorstellung, es könne sich bei der Unternehmung des Nikephoros um eine Tyrannis handeln; zu ähnlich gelagerten Fällen aus späterer Zeit s. etwa das das Beispiel Kaiser Michaels VIII. (dazu G. Prinzing, Ein Mann

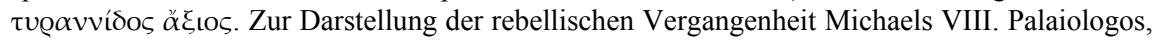


f) 43, 15-18: Die zuvor errungenen Siege ${ }^{85}$ sind der Erweis für die RechtmäBigkeit des eigenen Tuns, das von der göttlichen Vorsehung geleitet ist.

Natürlich lassen sich in auch diesem logos parakletikos die zu erwartenden Hauptkriterien bestimmen. Denn einen Nutzen zieht der zukünftige Herrscher selbst, aber mehr noch das gesamte Staatswesen, das sich in Gefahr befindet. Daneben handelt es sich bei der bevorstehenden Auseinandersetzung, um eine gute Sache, da sie von Gott gewollt ist, ${ }^{86}$ sowie, wenigstens für Leon Diakonos, um ein gerechtes und den allgemeinen Gesetzen entsprechendes Werk, da nicht Nikephoros die zuvor abgeschlossenen Vereinbarungen und Eide gebrochen habe, sondern sein Gegner Joseph Bringas. Daß aber Nikephoros nicht nur um das Wohl des Staates, sondern auch um sein eigenes bzw. das seiner Familie besorgt ist, gibt dem Vorgang bereits einen ironischen Beigeschmack und darf mit Blick auf das unrühmliche Ende des Nikephoros als Element der impliziten Kaiserkritik bewertet werden. Möglich wird der Erfolg nur mit der richtigen inneren Einstellung sein, und wenn es auch kein leichtes Unterfangen sein wird, so verspricht es doch Ruhm. Zuletzt wird der Erfolg als eine notwendige Folge eintreten, wobei Leon wieder eine eigenartige Doppelung zum Ausdruck bringt: Zum einen zielt Gott auf das durch den Eunuchen ${ }^{87}$ Joseph Bringas gefährdete Wohlergehen des Staates ab, zum anderen jedoch müssen Nikephoros Phokas und sein Heer erfolgreich sein, da sie im Falle des Scheiterns als Usurpatoren mit den entsprechenden

in: I. Vassis u.a. [Hrsgg.], Lesarten. Festschrift für Athanasios Kambylis zum 70. Geburtstag, dargebracht von Schülern, Kollegen und Freunden, Berlin/New York 1998, bes. 190-197).

85 Ebenso argumentierte Leon bereits in seiner ersten Rede (s. o. S. 114, Abschnitt c), was eindeutig gegen eine mögliche Historizität der Szene spricht.

86 Übrigens führt Hunger, Hochsprachliche profane Literatur (s. Anm. 5), I 370 in der Bewertung dieser Stelle in die Irre, da er im Begriff tò koعĩ tov (L.D., 43, 9) nur eine philosophischdistanzierende Umschreibung für Gott sah. In Wirklichkeit gelingt es Leon Diakonos auf diese Weise, den hier erforderlichen paränetischen Topos sogar expressis verbis in seinen Text einzufügen.

87 Leon Diakonos läßt in seinem Werk keine Gelegenheit aus, auf die fehlende Mannhaftigkeit und

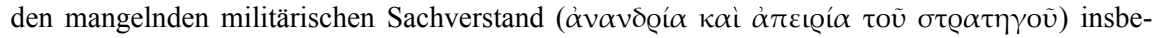
sondere der Eunuchen hinzuweisen, so bereits L.D., 6, 16-7, 8 im Zusammenhang mit der gescheiterten Kreta-Expedition des Konstantin Gongyles. Möglicherweise handelt es sich dabei jedoch um eine literarische Anspielung an Prokop von Kaisareia und seinen durch den Eunuchen Narses abgelösten „Helden“ Belisar (Proc. Caes., De bell., VIII 21, 6-7 [Veh]), doch kann dies hier nicht näher untersucht werden. Andererseits gibt sich Leon Diakonos auf diese Weise als Traditionalist zu erkennen, denn K. M. Ringrose, The Perfect Servant. Eunuchs and the Social Construction of Gender in Byzantium, Chicago/London 2003, 185-191 bringt eine Reihe von Beispielen dafür, daß das soziale Ansehen und die politischen Einflußmöglichkeiten der Eunuchen insbes. im 10. Jh. erheblich stiegen. Aber selbst jener Basileios Nothos, der zugunsten des Nikephoros gegen Joseph Bringas agierte, findet für unseren Autor (172, 14-17) schließlich doch den offenbar verdienten Tod durch ein göttliches Strafgericht. Mit Sicherheit führt es aber zu weit, kritische oder sarkastische Bemerkungen gegenüber Eunuchen als ein Charakteristikum volkstümlicher Literatur anzusehen, wie dies Hunger, Hochsprachliche profane Literatur (s.Anm. 5), I 258, tat. 
Folgen für ihr persönliches Wohlergehen zu rechnen haben. Dabei paßt das erste Element sehr wohl in den idealtypischen logos parakletikos, während das zweite wegen der angesprochenen individuellen Belange eher kritisch aufgenommen werden mußte. ${ }^{88}$ Immerhin schwächt Leon Diakonos diese Tendenz durch den wiederholten Hinweis darauf ab, daß das Heer seinen General zum Kaiser proklamiert hätte und er nur gezwungenermaßen und im Blick auf die allgemeinen politischen Umstände dazu bereit gewesen sei, das ihm angetragene Amt zu übernehmen.

Wie in den beiden vorangegangenen Fällen erfüllt die Rede ihren Zweck und bestärkte das anwesende Heer, ${ }^{89}$ das angesichts der Größe und der Gefahr der bevorstehenden Unternehmung jeden Mut hätte verlieren können, so sehr, daß einem Erfolg nichts mehr im Wege stand. Dabei war Nikephoros die Zuneigung seiner Soldaten sicher, denn er war einer der ihren, ${ }^{90}$ und zeichnete sich in erster Linie durch militärische Eigenschaften aus. Die im folgenden geschilderten Ereignisse zeigen, daß sich Nikephoros durchsetzen sollte, auch wenn es dabei selbstverständlich nicht zu der angedeuteten großen Schlacht um Konstantinopel kam. ${ }^{91}$ Gleichwohl steht die Rede am Endpunkt der Überlegungen des künftigen Kaisers, sich auf dieses Unternehmen einzulassen, das in der Darstellung des Leon Diakonos nur dann erfolgreich enden konnte, wenn er das Heer auf seine Seite zieht. Bezeichnenderweise zeigt sich gerade mit der allmählichen Abkehr vom soldatischen Leben, die sich bereits durch die von schlechten Ratgebern empfohlene Heirat mit der Witwe seines Vorgängers anzudeuten begann, ${ }^{92}$ ein erster Hinweis auf das bevorstehende bittere Ende des Nikephoros.

88 S. dazu bes. Luschnat, Feldherrnreden (s. Anm. 2), 123-125.

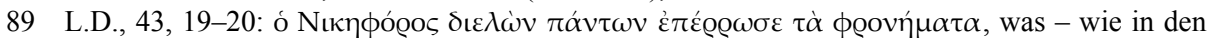
vorangehenden Fällen - den Erfolg der auf die Gesinnung eines jeden einzelnen abzielenden Paränese erkennen lassen soll. Gerade dies war der Zweck von Reden dieses Typus' (s. Albertus,

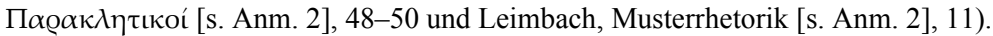

90 Literarisches und sprachliches Vorbild ist hier die alttestamentarische Schilderung Davids nach I Reg 17, 33.

91 Bereits Beck, Senat und Volk von Konstantinopel (s. Anm. 74), 33-34, wies darauf hin, daß mögliche Thronprätendenten im Falle einer Herrschaftsvakanz auf die Zustimmung der Bevölkerung Konstantinopels angewiesen waren. Eine „Eroberung“, konnte damit allenfalls auf rhetorisch-literarischem Wege stattfinden. Aufbauen ließ sich eine solche Fiktion auf der Grundlage des feierlichen Einzugs des Nikephoros in den Großen Palast. Dieser hatte ganz traditionell seinen Anfang am Goldenen Tor genommen, sodaß ein Literat diesem Ereignis einen imaginären „Kampf um Konstantinopel“ voranstellen konnte; zum Verlauf des Zugs s. Const. Porph., De Cer., I 96 (438,3-440,11 Reiske).

92 L.D., 49, 11-50, 1; vgl. damit auch Eus. Caes., Vita Const., I 19, 2 (Winkelmann). Für Leon Diakonos waren es Mönche, die den neuen Kaiser dazu überredeten, seine $\alpha \dot{\pi} \alpha \varrho \varepsilon \gamma \chi \varepsilon \dot{́} \emptyset \eta \tau$ $\sigma \omega \phi \varrho o \sigma u ́ v \eta$ aufzugeben und zu heiraten, wobei die Ehefrau als probates Mittel gegen eine uneingeschränkte Selbstherrschaft und -bestimmung verstanden wird. Nikephoros war jedoch schlechten Ratgebern gefolgt, denn an diesem Punkt (und noch nicht mit dem feierlichen Einzug in die Hauptstadt!) gibt Leon Diakonos das uneingeschränkt positive Bild auf, das er bislang 
Seine dritte und letzte Rede - die er nunmehr schon als Kaiser hielt - steht im Zusammenhang der Belagerung und späteren Eroberung der Stadt Antiochia am Orontes. Es läßt sich hier jedoch schon ein deutlicher inhaltlicher wie auch sachlicher Bruch wahrnehmen, der sich bereits mit der Erhebung des Nikephoros Phokas bzw. der Anerkennung als neuer Herrscher in Konstantinopel angedeutet hatte: Amt und Ehe beeinträchtigten ihn offenbar darin, diejenige Tätigkeit auszuüben, in der er sich am meisten auszeichnet, nämlich das asketisch-karge soldatische Leben. ${ }^{93}$ Und ein weiterer Schatten fiel auf Nikephoros: Seine Ehe stand unter dem Verdacht der Unrechtmäßigkeit, wobei sich Leon Diakonos nicht ausgesprochen darum bemüht, seinen Helden von diesem Vorwurf freizusprechen. ${ }^{94}$ So verwundert es nicht, daß sich in der Folge erste Niederlagen für Kaiser und Reich einstellten. Tarsos etwa konnte erst im zweiten Anlauf erobert werden, was Nikephoros Phokas in der Darstellung des Leon in einen heftigen inneren Konflikt geführt habe. ${ }^{95}$ Denn als Stratege sammelte er nur Siege ein, als Kaiser hingegen empfand er es als persönliche Herabwürdigung und Kränkung, an der Eroberung einer noch nicht einmal besonders gewaltigen Stadt gescheitert zu sein. Um diese Scharte auszuwetzen, besann er sich nun auf seine alte Stärke - und hatte schließlich Erfolg damit. ${ }^{96}$ Vor einem solchen Hintergrund versteht es sich

von seinem Helden gezeichnet hatte, ohne daß man gar an einen Erzähler- oder Quellenwechsel denken müßte, wie dies noch A. Р. Кaždan, Из истории византийской хронографии X в. II: Источники Лъва Диакона и Скилицы для истории третъей четверти X столетия, in: VV 20 (1961) 118-121 vorschlug. Übrigens entbehrt es durchaus nicht eines gewissen Reizes, dem siegreichen, strahlenden Helden des Leon Diakonos jenen „homo monstruosus“ gegenüberzustellen, dem man besser nicht zu nächtlicher Stunde begegnen sollte, wie Liudprand von Cremona Nikephoros II. eindrucksvoll beschreibt (s. Rel. de legat. Const., $3=188,44-57$ [Chiesa]); erg. dazu, wenn auch mit anderer Fragestellung, O. Kresten, Pallida mors Saracenorum (s. Anm. 42), 33.

93 L.D., 50, 14-20 könnte man auch folgendermaßen lesen: Kaiserliches Eigentum wird der Augusta Theophano geschenkt, und mit kaiserlichen Mitteln werden die Würdenträger und Reichen der Hauptstadt mit Schauspielen und Pferderennen den ganzen Winter über unterhalten.

94 L.D., 50, 1-14. Leon führt zwar die Argumentation des Kaisers gegen den öffentlich erhobenen Vorwurf der Verwandtschaftsehe an, doch schweigt er darüber, ob dieser seiner Meinung nach richtig ist oder nicht.

95 Bezeichnenderweise fehlen bei Leon Nachrichten über die Verhandlungen und Gesandtschaften bis zur Übergabe der Stadt Tarsos, über die die arabischen Quellen Auskunft geben (s. M. Canard, H'amdanides, 822-823 [s. Anm. 68], jetzt ausgeschrieben bei Dölger/Müller, Regesten I (s. Anm. 49), 1, 2, Nr. 706a-j). Leon Diakonos ist eben kein Historiograph im strengen Wortsinn - und will dies auch gar nicht sein. In diesem Sinn hat man es auch zu verstehen, daß er die erfolgreiche Rückeroberung der Insel Zypern durch den Patrikios Niketas Chalkutzes (Jo. Skyl., Hist., 270, 45-48 [Thurn]) nicht anführt, da sie nicht als Leistung des eigentlichen Helden in Anspruch genommen werden kann. Wäre sie - wie die folgende Sizilienexpedition - gescheitert, hätte Leon wie dort eine kaiserliche Mitschuld sicherlich nicht verschwiegen.

96 Das erste Scheitern vor Tarsos wird von Leon Diakonos ausführlich mit dem Hinwies darauf ironisiert, daß es sich ja nicht um einen ernst zu nehmenden Gegner gehandelt habe, sondern um eine überschaubare ( $\dot{v} \pi \varepsilon \rho i ́ \gamma \varrho \alpha \pi \tau o \varsigma)$, zudem in einer Ebene gelegene, für berittenen Einheiten leicht zugängliche Stadt (56,13-14). Folgerichtig verbringt Nikephoros den Winter vor der 
jedoch von selbst, daß Leon in die Rahmenhandlung dieser Episode keinen idealtypischen logos parakletikos einfügen konnte, da ein solcher bereits vor der ersten, vergeblichen Belagerung der Stadt hätte stehen müssen. Oder war das Schlachtenideal, wie es Leon Diakonos vermittelt, bereits so sehr gestört, daß der Kaiser und Feldherr gar die einen Erfolg garantierende Aufmunterungsrede vergessen hatte?

Für das Reich sollte es noch schlimmer kommen, denn die von Nikephoros im Jahr 964 veranlaßte Sizilien-Expedition scheiterte. ${ }^{97}$ Die Schuldigen sind für unseren Berichterstatter rasch gefunden, denn zum einen war der Befehlshaber des Heeres ein Eunuch, dem schon dadurch jedwedes mannhafte Verhalten abgesprochen werden konnte, ${ }^{98}$ während der Befehlshaber der Reiterei als eigenwilliger Hitzkopf abqualifiziert wird, der zu unüberlegten Handlungen neigte, und offenbar nur aufgrund seiner verwandtschaftlichen Beziehungen zum Kaiser mit dieser Aufgabe bedacht worden war. ${ }^{99}$ Nikephoros Phokas reagierte nach 966 auf die unvermeidliche Gefangennahme seiner beiden Offiziere angeblich mit einem bösen Drohbrief, dessen Wortlaut aber gewiß nicht der bei Leon wiedergegebene war. ${ }^{100}$ Den Brief in der von Leon Diakonos wiedergegeben Textfassung könnte man freilich als Platzhalter für eine jener Reden ansehen, um die es hier geht und außerdem als ein literarisches Konstrukt bewerten, das ein deutliches Ele-

zweiten Belagerung nicht im fernen, bequemen Konstantinopel, wo sich seine Leibgarde im Jahr zuvor allenfalls in Schattenkämpfen profilieren konnte (51, 3-4), sondern trifft nunmehr vor Ort und quasi im Feldlager die nötigen Vorbereitungen $(55,2-4)$. Dem gegenüber entbehrt es nicht einer gewissen Situationskomik, wenn ausgerechnet Nikephoros Phokas die vergebliche Belagerung Baris im Jahr 968 durch Otto I. als ein signifikantes Zeichen für die militärische Schwäche des Westkaisers bewertet (s. Rel. de legat. Const., $11=192,194-197$ [Chiesa]).

97 Dazu F. Maurici, Breve storia degli Arabi in Sicilia, Palermo 1995, 56-58, der den Bericht nach Leon Diakonos auswertet; s. ergänzend auch V. von Falkenhausen, Fonti italiane per il regno di Niceforo II Foca, in: G. Andenna/H. Houben (Hrsgg.), Mediterraneo, Mezzogiorno, Europa. Studi in onore di Cosimo Damiano Fonseca, Bari 2004, I 490-491, sowie Dölger/Müller, Regesten (s. Anm. 49), I, 1, 2, Nr. 699b (mit weiterer Literatur).

$98 \mathrm{Zu}$ den Vorbehalten Leons gegenüber den Eunuchen s. o. Anm. 87; zum Patrikios Niketas s. Ringrose, The Perfect Servant (s. Anm. 87), 137-138.

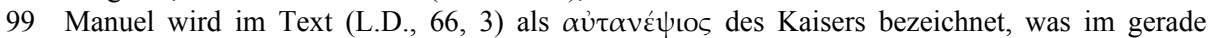
zitierten Regest 699b etwas unreflektiert mit Neffe übersetzt wurde; zum Verwandtschaftsgrad s. jetzt Talbot/Sullivan, History (s. Anm. 33), 115 Anm. 65, die zu Recht auf die Parallelquelle Jo. Skyl, Hist., 261, 10-12 (ed. Thurn) hinweisen. Die zur Charakterisierung Manuels gebrauchten Adjektive qualifizieren ihn eindeutig ab und erinnern an entsprechende Passagen bei Lukianos (Tim. 2, 6-8 und Phal. 1, 2, 6-7), wobei es sich auf jeden Fall um Eigenschaften handelt, die militärische Erfolge ausschließen. Die Kaiserkritik Leons besagt hier ein Doppeltes: Einerseits hätte sich Nikephoros besser selbst um die Expedition bekümmert, und wenn dies tatsächlich nicht möglich gewesen wäre, hätte er zumindest geeignete Generäle auswählen müssen. Wer also die Zeichen der Zeit nicht mehr erkennt, geht unaufhaltsam dem Untergang entgehen.

100 Dölger/Müller, Regesten (s. Anm. 49) I, 1, 2, Nr. 708. Leider äußert sich A. Beihammer, der Bearbeiter dieses Eintrags, nicht zur Frage der Historizität und nimmt den Wortlaut bei Leon Diakonos für bare Münze! 
ment der Kaiserkritik enthält. Denn während Nikephoros in früheren Zeiten erfolgreiche Schlachten selbst ausfocht, beschränkt er sich nunmehr darauf, einen Papierkrieg zu führen, und übersandte dem nur verbal heftig bedrohten aghlabidischen Emir von Tunis sogar noch das angeblich in den Syrienfeldzügen erbeutete Schwert Muhammads. ${ }^{101}$

Um die letzte Rede des Nikephoros Phokas einordnen zu können, war diese kurze Skizze der Vorgänge erforderlich, die sich bis zum Beginn der Belagerung Antiochias ereigneten, dem letzten Erfolg, an dem der Kaiser zumindest noch anfangs selbst beteiligt war. Sein bevorstehendes Ende wird dabei - ganz im apokalyptischen Gewand - durch eine Sonnenfinsternis angekündigt, die auch tatsächlich am 22. Dezember 968 stattfand. ${ }^{102}$ Nach Erfolgen in Edessa und im nördlichen Palästina ${ }^{103}$ stellte Nikephoros sein Heer nunmehr vor Antiochia auf, wo er scheinbar ganz unvermittelt eine Rede an seine Offiziere richtete und noch einmal persönlich in den Lauf der Dinge eingreift: ${ }^{104}$

a) 72, 23-73, 3: Mit göttlichem Beistand und der überragenden Tapferkeit des Heeres sei es gelungen, größere Erfolge gegen die Araber zu erzielen und sich in eine überlegene Position zu bringen. ${ }^{105}$

b) 73, 3-9: Der Garant des Erfolgs ist nur das Heer, das mit Entschlossenheit und wohlbedachter Stärke den göttlichen Plan umsetzt. ${ }^{106}$ Der General und Kaiser stellt dabei die Leistung seiner Person hinter die der Truppe zurück und stattet seinen Dank ab, wodurch der Text den Charakter eines (literarischen) Vermächtnisses erhält.

101 Dazu zuletzt Talbot/Sullivan, History (s. Anm. 33), 126 Anm. 5. Auch Prokop - und in einem Fall sogar Agathias - verwendeten die Rede in der Form von Gesandtenbriefen als literarisches Stilmittel (s. Taragna, Logoi historias [s. Anm. 22], 128-130), d.h. Leon Diakonos konnte hier durchaus auf entsprechende Vorbilder zurückgreifen.

102 L.D., 72, 1-18. Blendet man die biblische Dimension aus, in die diese Mitteilung gehört, könnte man darin durchaus ein sog. chronistisches Element im Sinne von Hunger (s. Anm. 5) und anderen sehen. In Wirklichkeit aber wird auf diese Weise die apokalyptische Spannung erhöht, die bereits durch die Erwähnung des schweren Erdbebens vom September 968 (68, 3-69, 3) und eines heftigen Sturzregen in Konstantinopel (69, 3-22) mit einem darauf folgenden Ascheregen $(69,22-70,2)$ aufgebaut wurde.

103 Ausführlich über diese Vorgänge s. G. Schlumberger, Un empereur byzantin au dixième siècle: Nicéphore Phocas, Paris 1890, bes. 718-723.

104 L.D., 72, 23-74, 12.

105 Die Paronomasie aus reóvot $\alpha(73,1)$ und menschlicher v́tóvot $\alpha(73,3)$ - wobei natürlich erstere obsiegt -, die bislang schon die Gewähr für die Siege der byzantinischen Truppen gegeben hatte, findet sich in einem durchaus vergleichbaren inhaltlichen Rahmen bereits bei Synes., De provid., II, 510, 1-8 (Garzya).

106 Es werden also wieder die traditionellen römischen Tugenden beschworen, die den Erfolg sichern; dazu s. etwa Polyb., Hist., 21, 22, 3-4 (Büttner-Wobst), aber auch Const. Porph., De legat., 243, 16-18 (Büttner-Wobst). 
c) 73, 9-11: Der Übergang auf die aktuelle Situation wird durch eine Anspielung auf den vom Redner behaupteten Wunsch des Heeres hergestellt, die belagerte Stadt Antiochia vollständig zu zerstören. ${ }^{107}$

d) 73, 11-18: Zwei Gründe sprechen gegen dieses Vorhaben, denn einerseits sei Antiochia die drittwichtigste Stadt der römischen Oikumene, ${ }^{108}$ andererseits begäbe sich das Heer dabei in eine unkalkulierbare Gefahr und könnte große Verluste erleiden.

e) 73, 18-74, 6: Die Aufgabe des Feldherrn ist es nun, dieses Dilemma aufzu-

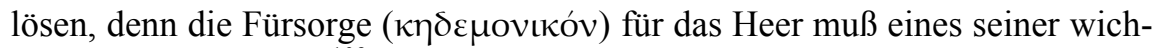
tigsten Anliegen sein. ${ }^{109}$ Daher folgt eine längere Paränese: Wer nicht mit einem möglichst geringen Aufwand den möglichst großen Erfolg sucht, erweist

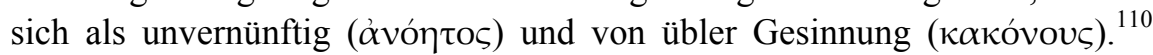
Außerdem brächte es weitere Gefahren, ein Gebiet zu zerstören, das ohnehin eigenes Land sei.

f) 74, 6-12: Der Weg zum Erfolg sei eine systematische Belagerung in Verbindung mit einer wohlüberlegten Guerillataktik, mittels derer man auf Dauer die Übergabe der Stadt erzwingen könne. ${ }^{11}$

Mit seiner Rede stellt der General und Kaiser als erstes heraus, daß der Krieg gegen Antiochia eine gute, von der göttlichen Vorsehung herbeigeführte Sache ist. Das Anliegen ist ein Gerechtes, da ohnehin römisch-byzantinisches Gebiet

107 Bei der angedeuteten Zerstörung handelt es sich natürlich nur um ein rhetorisches Moment, das die Dramatik der Situation unterstreichen soll; vgl. dazu Eus. Caes., Hist. eccl., VIII 12, 2 (Schwartz).

$108 \mathrm{Zu}$ den unterschiedlichen Forschungsansätzen, wie der hier genannte dritte Rang Antiochias zu erklären sei, s. jetzt Talbot/Sullivan, History (s. Anm. 33), 124 Anm. 104, die sich am ehesten dafür aussprechen, Antiochia als drittwichtigste Stadt des byzantinischen Reichs nach Konstantinopel und Thessalonike anzusehen. Der hier verwendete Begriff der Oikumene deutet jedoch eher auf einen kirchenpolitischen Zusammenhang hin, nämlich in Antiochia entweder das Ostpatriarchat mit dem dritten Rang zu sehen - oder gar noch den Vorrang vor Alexandria aufgrund der besonderen Bedeutung der Stadt im Rahmen der Entstehung der christlichen Kirche. Die erstgenannte Auffassung wird u.a. durch Iust., Nov. 109 pr., 518, 4-9 (Schoell/Kroll) gedeckt. Zum Thema sei verwiesen auf F. Gahbauer, Die Pentarchietheorie. Ein Modell der Kirchenleitung von den Anfängen bis zur Gegenwart (Frankfurter theologische Studien 42), Frankfurt am Main 1993, 71-74, der weitere Belege bringt. Übrigens berührt Nikephoros die für Antiochia durchaus wichtige Konfessions- und Religionsfrage nicht.

109 Man wird an die besänftigenden Worte bei Plut., Moralia, I, 133-20 = 66 E-F (Poh-

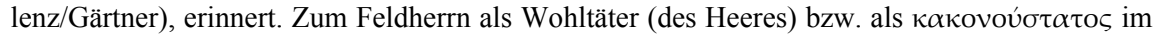
umgekehrten Falle s. Demosth., Or. 23, 6-7 (Blass). Aufgegriffen ist diese Stelle bei Herm., Inv., I, $1=99,14-21$ (Raabe), was dafür spricht, daß dieses Motiv auch in einem byzantinischen Text begegnen kann.

110 Zur Vorgehensweise des Kaisers vgl. Nik. II Phokas, De velitat., III-IV (Dagron), aber auch Demosth., Or. 23 (s. Anm. 109).

$111 \mathrm{Zu}$ den Maßnahmen im einzelnen sowie zu den Vorbereitungen, die noch der Kaiser bei der Ortswahl des byzantinischen Kastrons traf, vgl. Nik. II Phokas, De velitat., XXI (Dagron). 
eingenommen werde, und ein allgemeiner Nutzen wird dadurch gewahrt, daß der Kaiser sein Heer nicht über Gebühr größeren Gefahren aussetzen will. Und zuletzt sichert auch ein taktisch geführter Krieg den individuellen Ruhm.

Nikephoros Phokas leitet nach dieser Rede noch die für einen Guerillakrieg gegen Antiochia notwendigen Maßnahmen ein, um sodann nach Konstantinopel zurückzukehren. Damit weicht der Handlungsrahmen jedoch entscheidend von den früheren Berichten ab, nach denen der General selbst den bei Befolgung seiner Ratschläge als unabwendbar angekündigten byzantinischen Sieg erkämpfte. Als Kaiser oblagen ihm jedoch weitere Verpflichtungen, die ihn von dieser für Leon zentralen Aufgabe seines Amts abhielten.

Ruhe war ihm in der byzantinische Hauptstadt aber nicht vergönnt, denn die zuvor auf Veranlassung des Kaisers als Gegengewicht zu den Bulgaren zur Hilfe gerufene Kiever Rus' unter Svjatoslav Igorevič bedrohte nunmehr die Nordgrenze des Reichs. ${ }^{112}$ Offenbar eskalierte die Situation: der Herrscher kann nicht überall sein, und auch in der Hauptstadt selbst trachtete man ihm mittlerweile nach dem Leben. ${ }^{113}$ Allerdings sollte ihm noch der durch seine Rede vor Antiochia eingelei-

112 Dazu (nach wie vor unverzichtbar!) Schlumberger, Nicéphore Phocas (s. Anm. 103), 560-576 und 735-742; die frühere Literatur nur unkritisch ausgewertet hat P. Stephenson, Byzantium's Balkan Frontier. A Political Study of the Northern Balkans, 900-1204, Cambridge 2000, 48-51.

113 L.D., 85, 15-86, 6; Schlumberger, Nicéphore Phocas (s. Anm. 103), 745-760. Insgesamt schildert Leon Diakonos die Ereignisse der Ermordung des alten und die Erhebung des neuen Kaisers sehr emotionslos, wozu auch die detaillierte, aber sehr nüchterne Schilderung insbes. der Ermordung paßt. Allerdings wird im folgenden die Schuld des Joannes Tzimiskes offen genannt (z.B. 95, 5-19). Leon geht es dabei nicht so sehr um einen „krassen Naturalismus“ (Hunger, Hochsprachliche profane Literatur [s. Anm. 5], I 369), als vielmehr um eine zwangsläufige Abfolge der Ereignisse, wie sie die apokalyptische Konzeption seines Textes verlangt: Auf den siegreichen Reiter auf seinem weißen Pferd (s. L.D., 48, 1-3!), der zudem das Siegen im Namen trägt und dem die (Herrscher-)Krone (48, 19-20) gegeben wurde (s. Apk. 6, 1-2!), folgt ein Reiter auf einem feurigen Ross (auf einem solchen hielt übrigens auch Joannes Tzimiskes seinen Triumph in Konstantinopel nach seinem Sieg über die Rus' ab; s. L.D., 158, 14) und mit großen Waffen (vgl. dazu L.D., 132, 16), der vor allem den Unfrieden bringt (Apk. 6, 3-4). Außerdem heißt es im Bibeltext, die Menschen brächten sich zu seiner Zeit gegenseitig um, was man zum einen auf den Mord an Nikephoros anwenden kann, aber auch auf die Preisgabe seiner Mitverschwörer sowie der Kaiserin als Anstifterin gegenüber dem Patriarchen Polyeuktos. Interessanterweise erkannte für Leon Diakonos die Kaiserin Theophano in ihrem zweiten Ehemann Nikephoros auch den dritten apokalyptischen Reiter aus Apk. 6, 5-6, da sie bei ihm das Attribut der Waage als Symbol für eine allgemeine Teuerung sah, die angeblich in die Regierungszeit des Nikephoros gefallen sein soll $(63,24-64,12$; sicherlich ist es dabei kein Zufall, daß gerade dieser Einschub mit einer Prophezeiung endet, der Kaiser werde eines gewaltsamen Todes sterben). In denselben Kontext lassen sich auch seine Maßnahmen zur Geldentwertung (Schlumberger, Nicéphore Phocas [s. Anm. 103], 538-539) einordnen, von denen Leon Diakonos aber nicht spricht. Eine letzte Steigerung hätte hier die Figur des fahlen Reiters als Sinnbild des Todes nach Apk. 6, 8 bedeutet, aber das hätte zu einem inneren Widerspruch geführt, da dieser ja erst mit dem definitiven Ende der Zeiten auftreten darf. Sucht man nach literarischen Vorbildern, so steht der sprachlichen Komposition und Einordnung für die apokalyptischen Katastrophen zur Zeit von Justin I. und Justinian I. bei Prokop, die dort offen mit den beiden Kaisern in 
tete Erfolg vergönnt sein, denn die Stadt konnte durch seinen Sklaven Petros sowie durch den Patrikios Michael Burtzes tatsächlich eingenommen werden. ${ }^{114}$ Dabei war es der gute Rat des Nikephoros Phokas, der den Erfolg herbeiführte und für eine gewisse Zeit auch sichern sollte. Unmittelbar danach sollte es aber zur persönlichen Katastrophe für den Kaiser kommen, die in unserem Kontext jedoch keine Rolle spielt.

Einen letzten logos parakletikos setzt Leon Diakonos für Joannes Tzimiskes an, den Mörder und Nachfolger des Nikephoros Phokas. ${ }^{115}$ An der Bedrohung der Nordgrenze des byzantinischen Reichs hatte sich noch nichts geändert, zumal es Svjatoslav nach der Darstellung Leons gelungen war, die Bulgaren unter seine Botmäßigkeit zu zwingen. Als Gesandter nach Kiev hatte zunächst der Patrikios Kalokyres fungiert, der nach Auskunft Leons nunmehr selbst an der byzantinischen Kaiserwürde interessiert gewesen sein soll. ${ }^{116}$ Um so mehr mußte Tzimiskes darauf bedacht sein, hier einen Erfolg zu erzielen. Die Rede steht ganz zu Beginn der Unternehmung, die das byzantinische Heer in ein feindliches - zumindest in der Darstellung des Leon Diakonos -, massiv bedrohliches Umfeld führen soll, und wird daher nach der Passage des thrakischen Adrianopel in die Gegend jener „Kleisura“ plaziert, ${ }^{117}$ an der man das offenbar sichere Territorium

Verbindung gebracht wurden (s. M. Meier, Das andere Zeitalter Justinians. Kontingenzerfahrung und Kontingenzbewältigung im 6. Jahrhundert nach Chr. [Hypomnemata 147], Göttingen 2003, bes. 84-96) die zumindest vom Grundsatz her positive Bewertung der beiden Helden bei Leon gegenüber. Denn sie sind nur Werkzeuge des unausweichlichen eschatologischen Handelns Gottes, an dem sie jeweils durch ihre Person teilnehmen.

114 L.D., 81, 13-83, 1. Der dort berichtete Verlauf der Ereignisse stimmt nur partiell überein mit dem, was Jo. Skyl., Hist., 272, 91-273, 36 (Thurn) schildert. Dazu s. K.-P. Todt, Region und griechisch-orthodoxes Patriarchat von Antiocheia in mittelbyzantinischer Zeit und im Zeitalter der Kreuzzüge (969-1204). Teil I, Wiesbaden 2005, 189-193, der einen kritischen Vergleich der byzantinischen und arabischen Quellen bietet. Michael Burtzes wurde im Jahr 970 zum ersten byzantinischen Dux von Antiochia ernannt (ebd., 284-285 mit weiterer Literatur). Allgemein zur Situation nach Nikephoros II. Phokas s. auch C. Holmes, Byzantium's Eastern Frontier in the Tenth and Eleventh Centuries, in: D. Abulafia/N. Berend (Hrsgg.), Medieval Frontiers: Concepts and Practices, Aldershot 2002, bes. 87-88, 91-95 (für den hier behandelten Zeitraum).

115 L.D. 130, 19-132, 14. Grundsätzlich wäre gerade hier zu fragen, ob es sich dabei tatsächlich um einen parakletikos und nicht etwa um einen gewöhnlichen logos stratiotikos handelt. Allerdings überwiegt auch in diesem Textstück der mentale Zuspruch und die formal geforderte Ermahnung der Soldaten.

116 L.D., 77, 12-15 mit Jo. Skyl., Hist., 288, 1-5 (Thurn) und noch klarer bei Jo. Zon., Hist., 523 (Büttner-Wobst). Zum Patrikios Kalokyres sei hier nur verwiesen auf Talbot/Sullivan, History (s. Anm. 33), 111 Anm. 44, die auch die weitere Literatur zusammengetragen haben.

117 Dazu G. Schlumberger, L'épopée byzantine à la fin du dixième siècle. Partie 1: Guerres contre les Russes (...), Paris 1896 (ND Aalen 1969), 88-91. Adrianopel bezeichnet bei Leon Diakonos nur die Richtung, die das Heer genommen hat, ohne daß er - im Gegensatz zu Joannes Skylitzes - exakte Angaben zum weiteren Weg macht. Die Kleisura verwendet er hier aber nicht als ver-

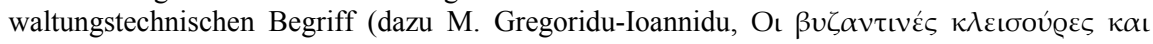

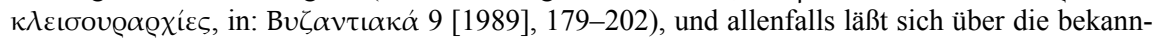
ten Gebirgspässe in den Rhodopen oder dem Balkan ermitteln (ebd., 200-201), welchen Weg 
verließ. Allein diese Tatsache birgt schon einen tiefgreifenden Unterschied zu den vorangegangenen Reden in sich: Auch in den bislang geschilderten Fällen bewegte sich das Heer in gefährlichen Regionen, doch wurden solche Gegebenheiten bei den siegreichen Phokaden erst gar nicht thematisiert. Dabei zeigte sich Tzimiskes gegenüber den bevorstehenden Ereignissen keineswegs unvorbereitet, weil er einen frühzeitigen Rückzug der Rus' über das Schwarze Meer durch eine Donausperre verhindern wollte. Eine weitere Diskrepanz liegt darin, daß die Rede des Tzimiskes aus zwei Teilen besteht, da es ihm im Gegensatz zu den Phokaden im ersten Anlauf nicht gelang, sein Heer zu überzeugen. Außerdem stellt Leon Diakonos heraus, daß es mit dem Übertritt in ein feindliches Gebiet für Tzimiskes und sein Heer um die persönliche Existenz geht, d.h. auch diese Rede steht an einer zentralen Stelle im skizzierten Handlungsverlauf:

$a_{1}$ ) 130, 19-23: Joannes Tzimiskes wird offensichtlich durch die von ihm vorgefunden Gegebenheiten überrascht. ${ }^{118}$ Spione berichteten ihm, daß der Augenblick günstig sei, die gefährlichen Pässe über das Balkan-Gebirge in das bulgarische Kerngebiet zu überqueren. ${ }^{119}$ Allerdings bestand keine Gewißheit darüber, ob die Gegner nicht doch Verteidigungsmaßnahmen getroffen hatten. Man kann dieser Aussage jedoch entnehmen, daß der Kaiser womöglich eine bestimmte Taktik verfolgte, wie man nach dem Erreichen der Grenze am besten gegen die Bulgaren operieren sollte. ${ }^{120}$

$\mathrm{b}_{1}$ ) 130, 23-131, 4: Die offenkundige Ursache für die günstige Ausgangsposition bestehe nach Ansicht des Tzimiskes darin, daß das Osterfest bevorstehe und die Bulgaren wohl deswegen nicht mit einer Invasion rechneten, weil die Byzantiner ihrer Meinung nach nicht auf die Freuden dieses Festes ${ }^{121}$ verzichten wollten. Natürlich wird später nicht gesagt, ob diese Hypothese zutraf.

das Heer nach Preslav nahm. Allerdings spielt dies auch keine Rolle, denn die Kleisura ist im Bericht Leons nur der Ort, an dem Joannes Tzimiskes für sich selbst sowie für das byzantinische Heer zu einer Entscheidung kommen muß. Die Topographie des Geländes ermöglicht zugleich einen vorläufigen dramatischen Höhepunkt im Text, der in seiner Tragweite den voranstehenden Reden durchaus entspricht.

118 Das Zögern des Kaisers und die nun in der Rede vorgetragenen sorgfältigen Überlegungen passen dabei sehr gut zur Konzeption des zweiten apokalyptischen Reiters (s. dazu o. Anm. 113)! Das Motiv der günstigen Gelegenheit, die es zu nutzen galt, fand sich bereits bei Prokop (s. Brodka, Geschichtsphilosophie [s. Anm. 20], 132-134 und 231).

119 Zur beschriebenen Vorgehensweise vgl. etwa Maur., Strat., VII A 3 (Dennis).

120 Offenbar ging Joannes Tzimiskes von Vorbereitungen des Gegners aus, wie sie z.B. in Nik. II Phok., De velitat. XXXIII 3-7 (Dagron) beschrieben werden, d.h. auch er rechnete wohl mit dem gezielten Einsatz bulgarischer Kundschafter.

121 Stephenson, Balkan Frontier (s. Anm. 112), 51, spricht davon, es sei ein erprobter strategischer Trick der Byzantiner gewesen, einen Angriff bereits in der Osterwoche zu beginnen, nennt aber keine weiteren Beispiele dafür. Allerdings sollte man sich fragen, inwieweit eine Kampagne mit einem durch das große Osterfasten geschwächte Heer nicht auch eine erhebliche Gefährdung der eigenen Seite bedeutet hätte. Was Leon mit dieser Stelle betonen will, ist vielmehr die 
$\left.c_{1}\right)$ 131, 4-8: Im Gegensatz zu den Phokaden, die ein weiteres Vorgehen klar bestimmten, äußert Joannes Tzimiskes gegenüber seinen Offizieren nur einen Vorschlag und sagt, was er für die beste Vorgehensweise hält, nämlich möglichst rasch und ohne großes Aufsehen die gefährliche Bergregion zu durchqueren.

$\left.d_{1}\right)$ 131, 8-12: Danach rät er zu einer Doppelstrategie, nämlich in einer ersten Auseinandersetzung die bulgarische Hauptstadt einzunehmen, um danach gegen die „Verwegenheit““122 der Rus’ vorzugehen. ${ }^{123}$

Der Vorschlag des Kaisers verfehlt jedoch sein Ziel, da die Offiziere darin eine zu große Gefahr für ihre Einheiten sehen und meinen, in der an sich ja qualitativ guten Zuversicht ihres Herrn die Unvernunft am Werke zu sehen. ${ }^{124}$ Daher stellt sich anstatt der zuvor sofort bekundeten Zustimmung ein längeres Schweigen ein, ${ }^{125}$ das Joannes Tzimiskes mit einer weiteren Rede beenden muß:

schlechte Meinung, die nach seiner Darstellung die Bulgaren von den „genußsüchtigen“ Byzantinern (L.D., 131, 2-4) hatten - was womöglich gar seinen eigenen Ansichten entsprach. Obwohl sich für die Makedonenkaiser durchaus überraschende Winterfeldzüge belegen lassen (Haldon, Warfare [s. Anm. 73], 54 mit Anm. 48 [mit Literatur]), sollte man bei dieser Stelle allenfalls an eine rhetorische Hyperbel denken, mit der der Redner die eigenen Truppen provozieren und in ihrem Eifer gegen den Feind bestärken möchte. Den historischen Hintergrund für dieses Zaudern bilden dabei sicherlich die schlechten Erfahrungen bei der Passage der BalkanPässe, die z.B. die Kaiser Konstantin IV. und Nikephoros I. gemacht hatten (dazu s. Schlumberger, L'épopée byzantine [s. Anm. 117], 92-93).

122 Leon verwendet hier den Begriff der $\dot{\alpha}$ Tóvot $\alpha$, der bei Thukyd., Hist., VII 67, 4 (= II, 200, 1620 [Hude]) in der Feldherrnrede des gegen die Athener auf Sizilien erfolgreich agierenden spartanischen Generals Gyllippos begegnet. Bezeichnet wird damit die mangelnde militärische Vorbereitung des Gegners, die den eigenen Sieg gewährleisten könne.

123 Leons Darstellung gibt sich damit als ex eventu verfaßte Prosopopoiie zu erkennen, da nun mit der vorgeschlagenen Abfolge Bulgaren/Preslav bzw. Kiever Rus'/Dorostolon der weitere Verlauf des schwierigen, letztendlich jedoch erfolgreichen Feldzugs vorgezeichnet wird. Dabei überwiegt eindeutig das Moment der großen Gefahr, in der sich das Heer des Tzimiskes befand, und im Gegensatz zu Nikephoros II. Phokas, den Leon niemals direkt für ein militärisches Scheitern verantwortlich macht, führt sein Nachfolger dieses Heer nunmehr in eine Unternehmung mit ungewissem Ausgang; zur chronologischen Abfolge und zur Dauer der Ereignisse s. F. Dölger, Die Chronologie des großen Feldzugs des Kaisers Johannes Tzimiskes gegen die Russen, in: BZ 32 (1932), 275-294, der aber Leon Diakonos wörtlich nimmt und an einem Beginn der Kampagne vor Ostern des Jahres 970 festhielt.

124 Die Zuhörer des Kaisers greifen damit im negativen Sinn gerade einen Begriff seiner Argumen-

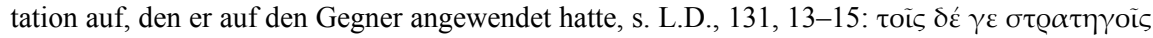

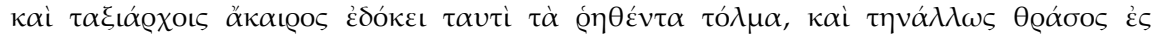

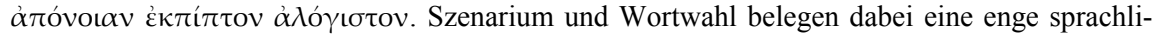
che Anlehnung an Diod. Sic., Bibl. hist., XIV 27, 1-5 (insbes. 27, 3, 1-7) (Dindorf/Fischer); zur Plausibilität eines solchen mittelbaren Zitats s. o. Anm. 41.

125 Die Redepause ist hier im Sinne von Rhet. graec., VIII, 411, nr. 52 (Walz) als demokratisches Element aus der Tradition der Polis zu verstehen, d.h. dem Heer sollte genug Zeit für eine Entscheidung gegeben werden, die dann jedoch ausbleibt. 
$\left.a_{2}\right)$ 131, 19-23: Der Skepsis des Heeres versucht Joannes Tzimiskes nunmehr seine Kriegserfahrung entgegenzuhalten. Er wisse sehr wohl, daß eigensinniges und verwegenes ${ }^{126}$ Handeln große Gefahr mit sich brächte, aber auch um die große Herausforderung für das Heer, die die kommenden Kämpfe bedeuteten. Aber eine günstige Gelegenheit zu nutzen ${ }^{127}$ müsse Motivation genug sein, die Furcht des Augenblicks zu überwinden.

$\mathrm{b}_{2}$ ) 131, 23-132, 7: Offenbar treffen hier nach Einschätzung des Kaisers - im Gegensatz zu den zuvor geschilderten Episoden - gleichwertige Heere aufeinander, wobei nur die einstweilen angenommene bzw. erspähte Sorglosigkeit $^{128}$ der Bulgaren den Byzantinern einen entscheidenden Vorteil verschaffen kann. Ließe man diese Gelegenheit verstreichen, wäre der gesamte Feldzug sinnlos.

$\mathrm{c}_{2}$ ) 132, 7-11: Der Kaiser ist sich der möglichen Gefahr bewußt, daß die Pässe doch von den Bulgaren bewacht sein könnten. Für diesen Fall sagt er sogar eine Niederlage des eigenen Heeres voraus. ${ }^{129}$

$\mathrm{d}_{2}$ ) 132, 11-14: Das Ende ähnelt sehr der ersten Rede des Nikephoros Phokas, ${ }^{130}$ da nun auch Joannes Tzimiskes seine Truppen dazu auffordert, sich im Kampf als Römer zu erweisen.

Sucht man nach den bekannten formalen Elementen des logos parakletikos, wird man hier nur partiell fündig. Der Zweck der Rede ist dabei eine mentale Stärkung, wie dies Tzimiskes am Ende auch ausgesprochen haben soll. Aber Leon Diakonos verzichtet bewußt auf einen Hinweis darauf, ob die Ansprache ihr Ziel beim Heer überhaupt erreicht. Einen Nutzen konnte allein der Kaiser feststellen, da er eine günstige Gelegenheit für den Angriff zu sehen glaubte, freilich ohne sich dessen sicher zu sein. Auch ließ es sich kaum als qualitativ gut bezeichnen, das ihm an-

126 Vgl. hiermit die entsprechenden Überlegungen des Nikias bei Thukyd., Hist., VII 8, 1-3 (= II, 154, 19-21 [Hude]), aber auch Xenoph., Anab., VI 5, 14 (Hude), die auch dieses retardierende Moment bei Leon Diakonos als literarische Fiktion erkennen lassen.

127 Zum hier verwendeten Motiv der „Entscheidung auf des Messers Schneide“ vgl. Hom., Il. 10, 173-174 und andere in Folge. Auf diese Weise dramatisiert Leon Diakonos seine Schilderung und erweckt den Eindruck, es käme nun unmittelbar zu einer alles entscheidenden Schlacht, obwohl sich die gesamte Kampagne mit wechselhaftem Kampferfolg noch über einige Monate hinziehen sollte. Allerdings hätte dann die Feldherrnrede an dieser Stelle keinen Sinn.

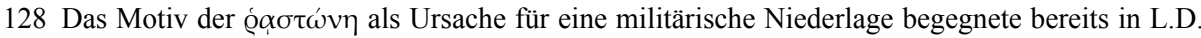
12, 22 (s. o. Anm. 34, sowie S. 123).

129 Auch hier handelt es sich um ein literarisches Motiv, das den abwägenden bzw. prüfenden Charakter unterstützt, den nach der Konzeption des Leon Diakonos Joannes Tzimiskes im Gegensatz zu seinem Vorgänger besessen haben soll (vgl. L. D., 94, 16; 102, 22-23); womöglich sollte man aber auch an das Motiv des dritten apokalyptischen Reiters aus Apk. 6, 5-6 denken (vgl. o. Anm. 113), das nunmehr für den alle möglichen Konsequenzen ,abwägenden“ Joannes Tzimiskes in Anspruch genommen wird.

130 L.D., 13, 3-10; s. dazu auch oben S. 114-115. 
vertraute Heer in eine größere Gefahr zu schicken, wodurch unter Umständen gar die sichere Existenz des Staates auf dem Spiel stünde. ${ }^{131}$ Somit bleibt es denn bei einem unbedingten Gehorsam, den Joannes Tzimiskes einfordert.

In logischer Konsequenz gestaltete Leon Diakonos nach Abschluß dieser letzten Rede auch ein anderes Szenarium als zuvor: Weder die Offiziere noch das Heer bekunden offen ihre Zustimmung, sondern sie folgen Tzimiskes als ihrem Feldherrn und Kaiser, ohne selbst von der Möglichkeit eines militärischen Erfolgs überzeugt zu sein. Folgerichtig führt Leon weiter aus, daß sich nunmehr der wohlgerüstete Kaiser auf sein Pferd setzte und offenbar willens war, allein gegen die Bulgaren bzw. die Truppen Svjatoslavs zu ziehen. Dabei ritt ihm nur seine Leibgarde, die Einheit der sogenannten „Unsterblichen““132 voran. Daß selbst der Kaiser trotz seines persönlichen Einsatzes nicht mit einer einfachen Passage des Balkan gerechnet hatte, läßt p. 132, 24-134, 1 erkennen: Denn erst nachdem man wider alle Erwartung ${ }^{133}$ das Gebirge hinter sich lassen konnte, beschleunigte das Heer seinen Marsch, um vor dem ersten Angriff auf Preslav an einer sicheren Stelle zu rasten und sich neu zu formieren. ${ }^{134}$

Die einzelnen Etappen der Auseinandersetzung mit den Bulgaren und den Truppen Svjatoslavs sieht Leon Diakonos dabei als eine Einheit. Nach schweren Kämpfen und auch Niederlagen für das byzantinische Heer sollte die Kampagne

131 Allerdings verwendet Leon Diakonos 132, 9-10 sehr wohl den Begriff des „Guten“, das sich nur für den Fall nicht zeigen würde, sollte das Heer dem Rat des Tzimiskes nicht folgen. Sicherlich muß man sich auch die Frage stellen, ob es sich bei diesem Textstück überhaupt um einen logos parakletikos handelt - oder etwa um einen symbuleutikos.

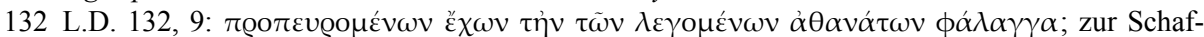
fung dieser Einheit s. auch 107, 11-13. Kenntnis über das historische Vorbild eines solchen Tagmas wird u.a. über Jo. Mal., Chron., 364, 11-12 (Thurn) vermittelt; zu den athanatoi s. weiterhin H.-J. Kühn, Die byzantinische Armee im 10. und 11. Jahrhundert. Studien zur Organisation der Tagmata (Byzantinische Geschichtsschreiber, Ergänzungsband 2), Wien 1991, 243-246. Allerdings entspricht die Aufstellung des an die Rede anschließenden Aufbruchs des Heeres tatsächlich den Regularien, wie sie sich in Maur., Strat., IX 4 (Dennis) für das Passieren von schwer zugänglichem Gelände und Engstellen finden. Zu den Pflichten des Feldherrn gehörte es dabei ebenso, daß er den richtigen Zeitpunkt (vgl. L.D., 132, 7) für den Beginn einer Unternehmung nicht etwa aus Furcht verstreichen lassen darf (Strat., VIII 1, 4). Womöglich sollte man aber doch die Myrmidonen des Achilleus (Hom. Il. 11, 790-805) als das literarische Vorbild der athanatoi des Tzimiskes sehen, die schon in der Spätantike als die "Seele“ der griechischen Truppen vor Troja bezeichnet wurden.

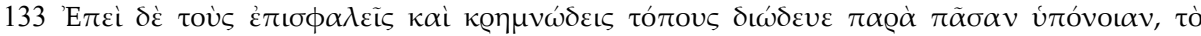

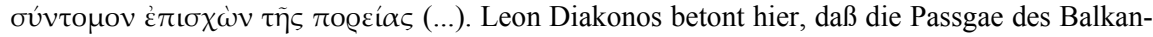
Gebirges wider alle Vernunft erfolgt sei, wobei natürlich auch der Kaiser in die negative Bewertung dieses Vorgangs einbezogen wird. Zum Sprachgebrauch und literarischem Vorbild s. auch Diod. Sic., Bibl. hist., XVI 31, 4-5 (Dindorf/Fischer).

134 Zur Errichtung eines Marschlagers auf einer Anhöhe mit gesicherter Wasserversorgung s. o. Anm. 111; im übrigen entspricht die Szene sogar den Anweisungen aus [Anon.], De re strat., XI 1-9 (Dennis) für die Errichtung einer Stadt. 
mit dem entscheidenden Sieg ${ }^{135}$ des Tzimiskes bei Dorostolon/Silistra ein glückliches Ende finden, das zuletzt nach dem plötzlichen Erscheinen eines heldenhaften weißen Reiters eintrat. ${ }^{136}$ Das neunte Buch Leons, das den endgültigen Sieg der Byzantiner zum Inhalt hat, endet mit einem für die literarische Ausgestaltung wichtigen Absatz, in dem das Eingangsmotiv des letzten logos parakletikos wieder aufgegriffen wird: ${ }^{137}$ Tzimiskes habe diesen Sieg aufgrund seiner reichen Kriegserfahrung sowie seiner rational-bedachtsamen Kühnheit ${ }^{138}$ errungen, woran sein Heer zunächst noch massive Zweifel hegte. Mehr Reden benötigt Leon Diakonos für sein Werk nicht. Zwar berichtet er in Buch X über weitere militärische Erfolge des Kaisers, doch war mit dem Sieg über Svjatoslav eine wichtige Vorbedingung für die Sicherung der Nordgrenze des byzantinischen Reichs geschaffen, nachdem zuvor Nikephoros Phokas im Osten für die entscheidenden Siege gesorgt hatte. ${ }^{139}$

135 L.D., 154, 23-155, 13. Die Angaben Leons über die Anzahl der gefallenen Waräger mit 15.500 gegenüber 350 gefallenen Rhomäern sind dabei als rhetorische Übertreibung zu bewerten, die die Größe des Siegs im $\alpha \hat{\gamma} \dot{\omega} v$ unterstreicht; s. auch Haldon, Byzantine Wars (s. Anm. 51), 104. Haldon bietet von 98-105 eine gründliche Analyse der Schlacht. Der Begriff des $\alpha \gamma \omega \dot{v} v$ begegnete übrigens schon in der Rede des Nikephoros vor seiner „Einnahme“ Konstantinopels.

136 L.D., 153, 22-154, 18. Nach Leon Diakonos deuten Kaiser und Heer diesen Reiter als Erscheinung des Soldatenheiligen Theodoros Stratelates, was in der Umbenennung von Dorostolon zu Theodorupolis $(158,1)$ zum Ausdruck kommt. Allerdings dürfte hierbei auch der apokalyptische Rahmen eine Rolle spielen, in dem der Bericht Leons steht. Denn nach Apk. 6, 1-2 bzw. 19, 11-16 ist es der Reiter auf dem Schimmel, der den Sieg in schwieriger Situation mit Sicherheit verheißt. Dafür spricht auch, daß der Paralleltext bei Jo. Skyl., Hist., 308, 10-309, 33 (Thurn) zwar weit ausführlicher über das angebliche Erscheinen des Heiligen spricht, ohne dabei aber das weiße Pferd zu erwähnen, das Leon nennt. Dölger, Chronologie (s. Anm. 123), 289-290, erschien diese Legende gar ,weit hergeholt“, da man den Heiligengedenktag für Theodoros allenfalls über das koptische Menologion für diesen Zeitraum veranschlagen könnte. Vielleicht sollte man darüber hinaus sogar eine bewußte Reminiszenz an Nikephoros II. Phokas in Betracht ziehen, den Leon Diakonos (40,1-3) ja auf einem Schimmel nach Konstantinopel einziehen ließ. Vgl. dazu auch M. Kintzinger, Der weiße Reiter. Formen internationaler Politik im Spätmittelalter, in: FMSt 37 (2003) 315-353, bes. 323-325 (mit Literatur). In starkem Kontrast dazu steht natürlich die Beschreibung des schnaubenden, unbändigen Pferdes, auf dem Nikephoros nach dem Bericht des Liudprand von Cremona geritten sein soll (s. Rel. de legat. Const., $23=197,375$ [Chiesa]) - auch wenn es sich dabei um eine Reminiszenz an die immer wieder verwendete Pferdeparabel aus Pl., Phaidr. 253e handelt, die den Kaiser selbst abqualifiziert.

137 L.D., 159, 2-9. Ein größerer Teil der in der kaiserlichen Eingangsrede vor Übertritt in feindliches Gebiet verwendeten Begriffe wie etwa der Mut, die Kühnheit oder das bedachte gegenüber dem selbstgefälligen Handeln, begegnet hier wiederum, freilich zu Gunsten der Rhomäer, wodurch Leon Diakonos einen angemessenen literarischen Abschluß seines Berichts erzielt.

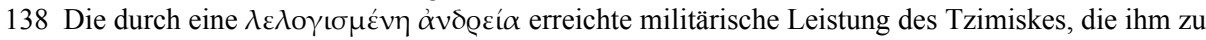
Beginn der Unternehmung von Seiten seiner Offiziere noch abgesprochen worden war $(131,19$ 21), erkennt Leon bei aller Kritik am Kaiser ohne Einschränkungen an.

139 Haldon, Warfare (s. Anm. 73), 40-41, weist zu Recht darauf hin, daß mit dem Sieg des Joannes Tzimiskes die entscheidende Vorbedingung für die Zerschlagung des bulgarischen Reichs durch Basileios II. geschaffen worden war. Zu den weiteren Erfolgen des Kaisers im Bereich der Ost- 
Welche Rückschlüsse kann man nun daraus ziehen, daß sich Leon Diakonos der rhetorischen Figur des logos parakletikos bediente? Sicherlich steht außer Zweifel, daß vor größeren Schlachten in der Tat auch Anfeuerungsreden gehalten wurden - zumal die militärtaktischen Handbücher den Nutzen solcher Reden ausdrücklich erwähnen. ${ }^{140}$ Konzeption und Wortwahl der einzelnen logoi lassen aber deutlich deren fiktiven Charakter erkennen, wobei der Gebrauch der wörtlichen Rede in sprachlicher Hinsicht einzig der dramatischen Ausgestaltung und Überhöhung des beschriebenen Szenariums dient. Am offensichtlichen Kulminationspunkt der Handlung sind es dabei immer Einsicht und Durchsetzungsvermögen des Feldherrn, die - wenn auch nach ganz bestimmten rhetorischen Regeln den militärischen Sieg des Heeres sichern. Trotz ihrer Kürze sind diese Reden inhaltlich eher Agathias ${ }^{141}$ nachempfunden als etwa Prokop, da sie nicht nur die Handlung gliedern, sondern das Für und Wider der jeweiligen Auseinandersetzung sorgfältig abwägen und auch in den Rahmen von übergeordneten politischen, juristischen oder philosophisch-praktischen Erwägungen stellen. Allerdings läßt sich ein klarer Unterschied für die beiden Phokaden auf der einen Seite und Joannes Tzimiskes auf der anderen ausmachen: Während nämlich erstere die Situation klar beherrschen, in der sie jeweils stehen, und sie das Heer ohne weiteres für sich gewinnen können, gelingt dies letzterem nicht ohne weiteres. Joannes Tzimiskes mußte sogar zweimal ansetzen, und selbst dadurch vermochte er sein Heer nicht zu überzeugen. Diesen Sachverhalt verdeutlichte Leon Diakonos schon zu Beginn seiner Schilderung des Bulgarienfeldzugs, da für ihn Joannes Tzimiskes offenbar keine klare Strategie verfolgte, sondern nur auf den unvorhersehbaren günstigen Moment vertraute, der sich dann auch tatsächlich einstellen sollte. Zuletzt war es aber das persönliche Beispiel des Kaisers sowie der Gehorsam seiner Truppen, die diese Kampagne des Jahres 970 überhaupt erst in Gang setzen.

grenze, wo sich das Zentrum der arabischen Macht mittlerweile jedoch in das fatimidische $\ddot{A}$ gypten verlagert hatte, s. Treadgold, History (s. Anm. 69), 511-512.

140 Maur., Strat., VII A 4 (Dennis); Nik. II. Phokas, De Velitat., XXIII $23-29$ (Dagron) u.a.m. In Zeiten einer zurückgehenden Redekultur und völlig geänderter militärischer Rahmenbedingungen mögen solche Ansprachen jedoch eher befremdlich erscheinen, s. dazu auch Hambsch, Feldherrnrede (s. Anm. 22), 235-236.

141 Dazu Taragna, Logoi historias (s. Anm. 22), 179-181. 
Abschließend sollte man sich fragen, welches literarische Genus Leon Diakonos mit seine Historia bediente. ${ }^{142}$ Eingangs hatte ich auf das oberflächliche Urteil Herbert Hungers hingewiesen, der von einer traditionellen „Geschichte“ mit chronographischen Elementen sprach. ${ }^{143}$ Dies wird dem Text aber keinesfalls gerecht, denn dann hätte es auch einer Darstellung etwa der intensiven Westpolitik oder des diplomatischen Austauschs mit Cordoba ${ }^{144}$ in jener Zeit bedurft. Die wiedergegebenen Ereignisse lassen somit eindeutig eine bestimmte Auswahl erkennen, die der Autor seinen Lesern respektive Hörern mitteilen wollte. Bringt man damit nun die verschiedenen erzählerischen Elemente wie den häufigen Gebrauch der wörtlichen Rede, die typisierende Beschreibung der Helden, die Reminiszenz an andere Begebenheiten, die den historischen Handlungsrahmen immer wieder - und dies scheinbar sinnlos - unterbrechen, oder etwa die apokalyptische Überhöhung der beiden Protagonisten in Verbindung, dann kommt man zu dem Schluß, daß es sich bei dem Text des Leon Diakonos um einen historiographischen Roman handelt, der nur bedingt objektivierbare Wahrheiten vermittelt, - und nur sehr eingeschränkt um ein Geschichtswerk im strengen Wortsinn. Ziel der Darstellung ist es - freilich nach dem Vorbild des Thukydides -, in erster Linie die besonderen persönlichen Leistungen und Erfolge der beiden zentralen Persönlichkeiten herauszustellen, über die vor allem berichtet wird. ${ }^{145}$ Etwaige Mißerfolge oder auch das für die Byzantiner in Relation zu den übrigen politischen Brennpunkten offenbar nicht ganz so wichtige politische Lavieren mit dem Westen passen nicht so recht ins Bild - mit der Folge, daß man generis causa darüber schweigen kann. Das rhetorische Stilmittel des logos parakletikos hat

142 E. Pietsch, Die Chronographia des Michael Psellos. Kaisergeschichte, Autobiographie und Apologie (Serta Graeca. Beiträge zur Erforschung griechischer Texte 20), Wiesbaden 2005, hat eines der Werke literarisch analysiert, das in klarer Folge zu Leon Diakonos steht. Auch wenn ihre Ergebnisse bereits zum großen Teil durch die entsprechenden Arbeiten J. N. Ljubarskijs zu Michael Psellos vorweggenommen wurden, so gelingt es ihr sehr wohl herauszuarbeiten, welche Probleme entstehen, will man das Werk für die Geschichtsschreibung im strengen Wortsinn retten. Die literarische Innovation, von der sie für Psellos spricht (z. B. 78-83 od. 131-133), ist in der Tat jedoch ein Verdienst des Leon Diakonos. Und wenn man sich über die Werktitel und Prooimien hinwegsetzt, wird man sicherlich beiden gerecht, wenn man sie heute als „Geschichtenschreiber" und nicht als (um Objektivität bemühte) Geschichtsschreiber auffaßt.

143 S. o. Anm. 8.

144 Dazu (in kritischer Auseinandersetzung mit den entsprechenden Einträgen bei Dölger/Müller, Regesten [s. Anm. 49], I, 1,2) J. Signes Codoñer, Bizancio y al-Ándalus en los siglos IX y X, in: I. Pérez Martín/P. Bádenas de la Peña (Hrsgg.), Bisanzio y la Península ibérica. De la Antiguëdad tardía a la Edad moderna (Nueva Roma 23), Madrid 2004, bes. 223-244.

145 Innerhalb eines solchen literarischen Konzepts verwundert es kaum, daß für Leon Diakonos bei der (angeblichen) Vergiftung des Herrschers wiederum - wie könnte es auch anders sein - ein Eunuch (L.D., 177, 4-5) seine ,schmuddeligen“ Hände im Spiel gehabt haben soll (zu diesem

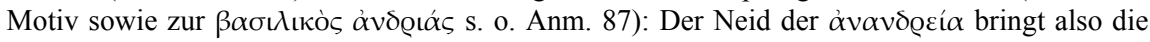

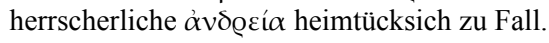


dabei eine doppelte Funktion: Zum einen wird die Bedeutung des persönlichen Einsatzes des Feldherrn bzw. Kaisers in einer für die eigene Seite sehr bedrohlichen Situation hervorgehoben, zum anderen markieren die im Vergleich zum thukydideïschen Vorbild sehr kurzen Reden einen Wendepunkt im Handlungsverlauf, da mit ihnen der spätere Sieg bereits vorweggenommen wird, und erweisen sich als literarische Konstruktionen. Formal steht das Werk damit, aber auch mit seiner sprachlichen Gestalt oder den zahlreichen, philologisch-antikisierenden Manierismen eindeutig in die Nähe von späteren Autoren wie Michael Psellos oder etwa der Alexias der Anna Komnene, die Jakuv N. Ljubarskij zu einem „Meisterwerk“ der byzantinischen Literatur erklärte. ${ }^{146}$ Wenn für eine solche Einschätzung allein der klassizistisch hohe Stil oder die Kenntnis und der Gebrauch von Homer oder anderen antiken Autoren ausreichen sollten, dann hätte man diese entgegen der allgemein verbreiteten Bewertung auch auf Leon Diakonos anzuwenden. ${ }^{147}$

$\mathrm{Ob}$ dem tatsächlich so ist, müssen weitere stilistische und rhetorische Untersuchungen aufzeigen. Den Text und vor allem auch die qualitativen Urteile, die er vermitteln will, darf man sicherlich nur bedingt wörtlich nehmen. Er ist damit ein sehr gutes Beispiel für das von Paul Magdalino angekündigte literaturwissenschaftliche Instrument, mit dem eine ganze Reihe von historiographischen Texten aus der byzantinischen Zeit erst einmal für das Verständnis moderner Historiker aufbereitet werden sollten, ${ }^{148}$ bevor man einzelnen dieser Autoren etwa Ungenauigkeiten im Detail oder gar Fehler zum Vorwurf macht, die sich durch die Konzeption und die literarische Anlage eines solchen Werkes erklären - und für den Autor unerheblich gewesen sein dürfen. Gerade die Analyse rhetorischer Figuren und Stilmittel wird dabei einen nicht zu unterschätzenden Stellenwert besitzen: So

146 S. dazu N. J. Ljubarskij, Why is the 〈Alexiad〉 a masterpiece of Byzantine Literature?, in: Th. Gouma-Peterson (Hrsg.), Anna Komnene and Her Times, New York/London 2000, bes. 170

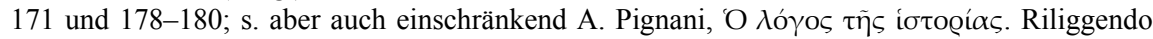
Anna Comnena, in: Criscuolo/Maisano (Hrsgg.), Categorie linguistiche e concettuali della storiografia bizantina (s. Anm. 1), 149-158, die das Werk Annas sicherlich zutreffend in den Bereich der Hofrhetorik versetzt und für die literarischen Genera Enkomion und Monodie in Anspruch nimmt.

147 S. o. Anm. 7.

$148 \mathrm{Zu}$ Magdalino s. o. Anm. 6 (Pietsch, Psellos [s. Anm. 142] meint mit Seite 133 zwar auch den Beitrag P. Magdalinos, verweist aber pauschal auf den gesamten Tagungsband). Übrigens hat D. Roques, Histoire et rhétoriques dans l'œuvre de Procope de Césarée: Procope est-il un historien?, in: Criscuolo/Maisano (Hrsgg.), Categorie linguistiche e concettuali della storiografia bizantina (s. Anm. 1), 3-39, in seiner vorzüglichen Studie für Prokop von Kaisareia zeigen können, daß der Gebrauch von antiken Versatzstücken und Übernahmen aus den literarischen Vorbildern seine historiographische Zuverlässigkeit doch erheblich einschränken. Um so mehr sollte man diese Erkenntnis auch auf die Epigonen Prokops anwenden, zu denen Leon Diakonos trotz seiner Nähe zu Agathias zweifelsohne zählt. Zum Verhältnis von Prokop und Agathias, den Hase als Vorbild für Leon heranzog (s. o. Anm. 6), s. bei Roques, ibid., bes. 36-39. 
mancher Schlachtenschilderung wird man noch ihren rhetorisch-literarischen Idealtypus gegenüberstellen müssen, wie ihn einst Hermogenes und Theon von Alexandreia formuliert hatten. ${ }^{149}$

149 Hermog., prog. 23,1-6 (Rabe); Theon Al., Prog. 119, 14-21 (Spengel). 
